\title{
Trinomials, singular moduli and Riffaut's conjecture
}

\author{
Yuri Bilu, Florian Luca, Amalia Pizarro-Madariaga ${ }^{\mathrm{c}}$
}

August 30, 2021

\begin{abstract}
Riffaut 22] conjectured that a singular modulus of degree $h \geq 3$ cannot be a root of a trinomial with rational coefficients. We show that this conjecture follows from the GRH and obtain partial unconditional results.
\end{abstract}

\section{Contents}

1 Introduction 1

2 Generalities on singular moduli

3 Suitable integers 5

4 Roots of trinomials and the principal inequality 8

5 Suitable integers for trinomial discriminants 11

6 Small discriminants 13

7 Structure of trinomial discriminants

8 Primality of suitable integers $\quad 24$

9 A conditional result 25

10 Bounding all but one trinomial discriminants $\quad 29$

11 The quantities $h(\Delta), \rho(\Delta)$ and $N(\Delta)$

12 The signature theorem

\section{Introduction}

A singular modulus is the $j$-invariant of an elliptic curve with complex multiplication. Given a singular modulus $x$ we denote by $\Delta_{x}$ the discriminant of the associated imaginary quadratic order. We denote by $h(\Delta)$ the class number of

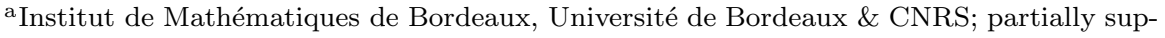
ported by the MEC CONICYT Project PAI80160038 (Chile), and by the SPARC Project P445 (India)

${ }^{\mathrm{b}}$ School of Mathematics, University of the Witwatersrand; Research Group in Algebraic Structures and Applications, King Abdulaziz University, Jeddah; Centro de Ciencias Matematicas, UNAM, Morelia; partially supported by the CNRS "Postes rouges" program

${ }^{c}$ Instituto de Matemáticas, Universidad de Valparaíso; partially supported by the Ecos / CONICYT project ECOS170022 and by the MATH-AmSud project NT-ACRT 20-MATH-06
} 
the imaginary quadratic order of discriminant $\Delta$. Recall that two singular moduli $x$ and $y$ are conjugate over $\mathbb{Q}$ if and only if $\Delta_{x}=\Delta_{y}$, and that there are $h(\Delta)$ singular moduli of a given discriminant $\Delta$. In particular, $[\mathbb{Q}(x): \mathbb{Q}]=h\left(\Delta_{x}\right)$. For all details, see, for instance, [12, $\S 7$ and $\S 11]$.

In this article we study the following question.

Problem 1.1. Can a singular modulus of degree $h \geq 3$ be a root of a trinomial with rational coefficients?

Here and below a trinomial is an abbreviation for a monic trinomial nonvanishing at 0 ; in other words, a polynomial of the form $t^{m}+A t^{n}+B$ with $m>n>0$ and $B \neq 0$.

This problem emerged in the work of Riffaut 22] on the effective AndréOort conjecture. We invite the reader to consult the article of Riffaut for more context and motivation. Riffaut conjectured that the answer is negative, but, as he admits, "much about trinomials is known, but this knowledge is still insufficient to rule out such a possibility".

We believe, however, that the problem is motivated on its own, independently of Riffaut's work, because it is of interest to learn more about the relationship between two very classical objects like rational trinomials and singular moduli.

In Section 9 we prove that Riffaut's conjecture follows from the GRH.

Theorem 1.2. Assume the Generalized Riemann Hypothesis for the Dirichlet $L$-functions. Then a singular modulus of degree at least 3 cannot be a root of a trinomial with rational coefficients.

We also obtain some partial unconditional results. To state them, we have to introduce some definitions that will be used throughout the article.

Let $\Delta$ be an imaginary quadratic discriminant. We call $\Delta$ trinomial discriminant if $h(\Delta) \geq 3$ and the singular moduli of discriminant $\Delta$ are roots of a trinomial with rational coefficients. If this trinomial is of the form $t^{m}+A t^{n}+B$ then we say that $\Delta$ is a trinomial discriminant of signature $(m, n)$.

Note that a trinomial discriminant may, a priori, admit several signatures. However, there can be at most finitely many of them, and all of them can be effectively computed in terms of $\Delta$. This follows from the results of article [3] and the following property: for any singular modulus $x$ and positive integer $k$ we have $\mathbb{Q}\left(x^{k}\right)=\mathbb{Q}(x)$, see [22, Lemma 2.6].

Now we are ready to state our unconditional results. First of all, in Sections 6 and 10 we show that a trinomial discriminant cannot be too small, and, with at most one exception, cannot be too large either.

Theorem 1.3. Every trinomial discriminant $\Delta$ satisfies $|\Delta|>10^{11}$, and at most one trinomial discriminant $\Delta$ satisfies $|\Delta| \geq 10^{160}$. In particular, the set of trinomial discriminants is finite. form.

Next, in Section 7 we show that trinomial discriminants are of rather special 
Theorem 1.4. Every trinomial discriminant is of the form $-p$ or $-p q$, where $p$ and $q$ are distinct odd prime numbers. In particular, trinomial discriminants are odd and fundamental.

Finally, in Section 12 we show that trinomials vanishing at singular moduli are themselves quite special.

Theorem 1.5. Let $\Delta$ be a trinomial discriminant of signature $(m, n)$. Assume that $|\Delta| \geq 10^{40}$. Then $m-n \leq 2$.

Plan of the article. In Section 2 we remind general facts about singular moduli, to be used throughout the article. In Section 3 we introduce and study the basic notion of suitable integer. A positive integer $a$ is called suitable for a discriminant $\Delta$ if there exists $b \in \mathbb{Z}$ such that $b^{2} \equiv \Delta \bmod 4 a$ and $(b+\sqrt{\Delta}) / 2 a$ belongs to the standard fundamental domain (plus a certain coprimality condition must be satisfied). We give various recipes for detecting suitable integers of arbitrary discriminants, so far without any reference to trinomials.

In Section 4 we obtain some metrical properties of roots of trinomials, both in the complex and non-archimedean setting. Applying them to singular moduli that are roots of a trinomial, we obtain the "principal inequality", a basic tool instrumental for the rest of the article. In Section 5 we use the "principal inequality" to study suitable integers of trinomial discriminants: they turn out to be very large, of order of magnitude $|\Delta|^{1 / 2} / \log |\Delta|$, and densely spaced.

In Section 6 we show that trinomial discriminants cannot be too small (the first statement of Theorem 1.3). The argument uses the results of the previous sections and computations with PARI [26] and SAGE [27.

In Section 7 we prove Theorem 1.4 on the structure of trinomial discriminants, using careful analysis of suitable integers. In the follow-up Section 8 we show that suitable integers of trinomial discriminants are prime numbers.

In Section 9 we obtain the conditional result (Theorem 1.2) and in Section 10 we obtain an unconditional upper bound for all but one trinomial discriminant (the second statement of Theorem 1.3). The principal arguments of these sections already appeared elsewhere 15, 17, 21, and we only had to adapt them to our situation.

In Section 11 we study the class number and other numerical characteristics of trinomial discriminants. Using the results from that section, we prove Theorem 1.5 in Section 12

Acknowledgments. We thank Michael Filaseta, Andrew Granville, Sanoli Gun, Tanmay Khale, Chazad Movahhedi, Olivier Ramaré, Igor Shparlinski and the mathoverflow user Lucia for helpful suggestions.

We are most grateful to the anonymous referees, who corrected several mistakes, and made many very useful comments that helped us to improve the presentation.

All calculations were performed using PARI 26] or SAGE [27. We thank Bill Allombert and Karim Belabas for the PARI tutorial. The reader may consult 
https://github.com/yuribilu/trinomials to view the PARI script used for this article.

Yuri Bilu thanks the University of Valparaiso and the Institute of Mathematical Sciences (Chennai) for stimulating working conditions.

Florian Luca worked on this project during visits to the Institute of Mathematics of Bordeaux from March to June 2019, and to the Max Planck Institute for Mathematics in Bonn from September 2019 to February 2020. He thanks these institutions for hospitality and support.

Amalia Pizarro-Madariaga thanks the Max Planck Institute for Mathematics in Bonn for hospitality and stimulating working conditions.

\subsection{Some general conventions}

Throughout the article we use $O_{1}(\cdot)$ as a quantitative version of the familiar $O(\cdot)$ notation: $X=O_{1}(Y)$ means that $|X| \leq Y$.

We denote by $(\Delta / p)$ the Kronecker symbol. The general definition of the Kronecker symbol can be found, for instance, on page 202 of [14. In this article, however, we will always use it in the special case when $\Delta$ is a discriminant (and, in particular, $\Delta \equiv 0,1 \bmod 4)$ and $p$ is a prime number. In this case $(\Delta / p)$ is just the Legendre symbol $\bmod p$ if $p$ is an odd prime, and

$$
\left(\frac{\Delta}{2}\right)= \begin{cases}1, & \Delta \equiv 1 \bmod 8 \\ -1, & \Delta \equiv 5 \bmod 8 \\ 0, & \Delta \equiv 0 \bmod 4 .\end{cases}
$$

We use the standard notation $\omega(\cdot)$ for the number of prime divisors (counted without multiplicities). If $\mathfrak{p}$ is a prime of a number field then we denote $\nu_{\mathfrak{p}}(\cdot)$ the $\mathfrak{p}$-adic valuation (normalized so that its group of values is $\mathbb{Z}$ ).

\section{Generalities on singular moduli}

In this section we summarize some properties of singular moduli used in the article. Unless the contrary is stated explicitly, everywhere below the letter $\Delta$ stands for an imaginary quadratic discriminant; that is, $\Delta<0$ and $\Delta \equiv 0,1 \bmod 4$.

Denote by $\mathcal{F}$ the standard fundamental domain: the open hyperbolic triangle with vertices

$$
\zeta_{3}=\frac{-1+\sqrt{-3}}{2}, \quad \zeta_{6}=\frac{1+\sqrt{-3}}{2}, \quad i \infty,
$$

together with the geodesics $\left[i, \zeta_{6}\right]$ and $\left[\zeta_{6}, \infty\right]$. It is well-known (see, for instance, [4, Proposition 2.5] and the references therein) that there is a one-to-one correspondence between the singular moduli of discriminant $\Delta$ and the set $T_{\Delta}$ of triples $(a, b, c)$ of integers with $\operatorname{gcd}(a, b, c)=1$, satisfying $b^{2}-4 a c=\Delta$ and

$$
\text { either }-a<b \leq a<c \text { or } 0 \leq b \leq a=c .
$$


If $(a, b, c) \in T_{\Delta}$ then $(b+\sqrt{\Delta}) / 2 a$ belongs to $\mathcal{F}$, and the corresponding singular modulus is $j((b+\sqrt{\Delta}) / 2 a)$.

We call a singular modulus dominant if in the corresponding triple $(a, b, c)$ we have $a=1$. For every $\Delta$ there exists exactly one dominant singular modulus of discriminant $\Delta$.

The inequality

$$
|| j(z)\left|-e^{2 \pi \operatorname{Im} z}\right| \leq 2079
$$

holds true for every $z \in \mathcal{F}$; see, for instance, [5, Lemma 1]. In particular, if $x$ is a singular modulus of discriminant $\Delta$ corresponding to the triple $(a, b, c) \in T_{\Delta}$ then

$$
|| x\left|-e^{\pi|\Delta|^{1 / 2} / a}\right| \leq 2079
$$

This implies that

$$
\begin{array}{ll}
|x| \leq e^{\pi|\Delta|^{1 / 2}}+2079 & \text { in any case; } \\
|x| \geq e^{\pi|\Delta|^{1 / 2}}-2079 & \text { if } x \text { is dominant; } \\
|x| \leq e^{\pi|\Delta|^{1 / 2} / 2}+2079 & \text { if } x \text { is not dominant. }
\end{array}
$$

These inequalities will be systematically used in the sequel, sometimes without special reference.

\section{$3 \quad$ Suitable integers}

Everywhere in this section $\Delta$ is an imaginary quadratic discriminant and $a$ a positive integer.

Call an integer a suitable for $\Delta$ if there exist $b, c \in \mathbb{Z}$ such that $(a, b, c) \in T_{\Delta}$. Note that 1 is always suitable, and that a suitable $a$ satisfies $|\Delta| \geq 3 a^{2}$ : this follows from the fact that $(b+\sqrt{\Delta}) / 2 a$ belongs to the standard fundamental domain, or directly from the relation $\Delta=b^{2}-4 a c$ and the inequalities $|b| \leq a \leq c$. Moreover, equality $|\Delta|=3 a^{2}$ is possible only when $\Delta=-3$ and $a=1$, and we have the strict inequality $|\Delta|>3 a^{2}$ when $\Delta \neq-3$.

In the following proposition we collect some useful tools for detecting suitable integers.

Proposition 3.1. 1. Assume that $\operatorname{gcd}(a, \Delta)=1$, that $\Delta$ is a square $\bmod 4 a$, and that $|\Delta| \geq 4 a^{2}$. Then a is suitable for $\Delta$.

2. Let $a$ be suitable for $\Delta$ and $a^{\prime}$ a divisor of a such that $\operatorname{gcd}\left(a^{\prime}, \Delta\right)=1$. Then $a^{\prime}$ is suitable for $\Delta$ as well.

3. Let $p$ be a prime number satisfying $(\Delta / p)=1$ and $|\Delta| \geq 4 p^{2}$. Then $p$ is suitable for $\Delta$.

4. Assume that $\Delta$ is even, that $\Delta \not \equiv 4 \bmod 32$, and that $|\Delta| \geq 76$. Then 2 or 4 is suitable for $\Delta$. 
5. Assume that $\Delta \equiv 4 \bmod 32$. Let $k \geq 3$ be an integer such that $|\Delta| \geq 2^{2 k+2}$. Then $2^{k}$ is suitable for $\Delta$. In particular, if $|\Delta| \geq 2^{10}$ then 8 and 16 are suitable for $\Delta$.

6. Assume that $\Delta=-2^{\nu} a a^{\prime}$, where $\nu=\nu_{2}(\Delta)$ and $a, a^{\prime}$ are positive odd integers with $\operatorname{gcd}\left(a, a^{\prime}\right)=1$. Then $\min \left\{a, a^{\prime},\left(a+a^{\prime}\right) / 4\right\}$ is suitable for $\Delta$ if $\Delta$ is odd, and $\min \left\{a, a^{\prime}\right\}$ is suitable if $\Delta$ is even.

The proof requires a simple lemma, telling that $0^{2}, 1^{2}, \ldots, m^{2}$ exhaust all squares $\bmod 4 m$.

Lemma 3.2. Let $m$ be a positive integer and $x$ an integer. Then there exists an integer $y$ satisfying $0 \leq y \leq m$ and $y^{2} \equiv x^{2} \bmod 4 m$.

Proof. Since $x_{1} \equiv x_{2} \bmod 2 m$ implies that $x_{1}^{2} \equiv x_{2}^{2} \bmod 4 m$ we may assume that $-m<x \leq m$. Now set $y=|x|$.

Proof of Proposition 3.1. 1. If $\Delta$ is a square $\bmod 4 a$ then Lemma 3.2 produces $b \in \mathbb{Z}$ satisfying $0 \leq b \leq a$ and $\Delta \equiv b^{2} \bmod 4 a$. If $\operatorname{gcd}(a, \Delta)=1$ then we have $\operatorname{gcd}(a, b)=\overline{1}$, and $\left(a, b,\left(b^{2}-\Delta\right) / 4 a\right) \in T_{\Delta}$ when $|\Delta| \geq 4 a^{2}$. This proves item 1 .

2. If $a$ is suitable for $\Delta$ then $|\Delta| \geq 3 a^{2}$ and $\Delta$ is a square $\bmod 4 a$. If $a^{\prime}$ is a proper divisor of $a$ then $|\Delta| \geq 12\left(a^{\prime}\right)^{2}$ and $\Delta$ is a square $\bmod 4 a^{\prime}$. Hence item 2 follows from item 1 .

3. Let $p$ a prime number ( $p=2$ included). Then the condition $(\Delta / p)=1$ implies that $\Delta$ is a square $\bmod 4 p$ and is co-prime with $p$. Hence item 3 follows from item 1 as well.

4. When $16 \mid \Delta$, select $b \in\{0,4\}$ to satisfy $\nu_{2}\left(\Delta-b^{2}\right)=4$. Then we have $\left(4, b,\left(b^{2}-\Delta\right) / 16\right) \in T_{\Delta}$ provided that $|\Delta| \geq 64$. Furthermore,

$$
\begin{aligned}
(2,0,-\Delta / 8) & \in T_{\Delta} & & \text { if } \Delta \equiv 8 \bmod 16 \text { and }|\Delta| \geq 24, \\
(2,2,(4-\Delta) / 8) & \in T_{\Delta} & & \text { if } \Delta \equiv 12 \bmod 16 \text { and }|\Delta| \geq 20, \\
(4,2,(4-\Delta) / 16) & \in T_{\Delta} & & \text { if } \Delta \equiv 20 \bmod 32 \text { and }|\Delta| \geq 76 .
\end{aligned}
$$

This proves item 4

5. If $\Delta \equiv 4 \bmod 32$ then, by Hensel's lemma, for $k=3,4, \ldots$ there exists $x_{k} \in \mathbb{Z}$ such that $\Delta / 4 \equiv x_{k}^{2} \bmod 2^{k}$. Hence, setting $b_{k}=2 x_{k}$, we find $b_{k} \in \mathbb{Z}$ with the property $\Delta \equiv b_{k}^{2} \bmod 2^{k+2}$. Moreover, Lemma 3.2 implies that $b_{k}$ can be chosen to satisfy $0 \leq b_{k} \leq 2^{k}$. Note also that $\nu_{2}\left(b_{k}\right)=1$, which implies that $\nu_{2}\left(b_{k}^{2}-\left(2^{k}-b_{k}\right)^{2}\right)=k+2$. Hence, replacing (if necessary) $b_{k}$ by $2^{k}-b_{k}$ we may assume that $\nu_{2}\left(b_{k}^{2}-\Delta\right)=k+2$. Then $\left(2^{k}, b_{k},\left(b_{k}^{2}-\Delta\right) / 2^{k+2}\right) \in T_{\Delta}$ provided $|\Delta| \geq 2^{2 k+2}$. This proves item 5 , 
6. In item 6 we will assume that $a^{\prime} \geq a$. Then for odd $\Delta$ we have

$$
\begin{aligned}
\left(a, a, \frac{a+a^{\prime}}{4}\right) & \in T_{\Delta} & & \text { when } a^{\prime} \geq 3 a, \\
\left(\frac{a+a^{\prime}}{4}, \frac{a^{\prime}-a}{2}, \frac{a+a^{\prime}}{4}\right) & \in T_{\Delta} & \text { when } a & \leq a^{\prime} \leq 3 a,
\end{aligned}
$$

and for even $\Delta$ we have $\left(a, 0,2^{\nu-2} a^{\prime}\right) \in T_{\Delta}$. This proves item 6 .

We want to extend item 6 to the case when $a$ and $\Delta / a$ are not coprime. This is possible in the special case when $a$ is an odd prime power with even exponent.

Proposition 3.3. Let $p$ be an odd prime number and $k$ a positive integer such that $p^{2 k+1} \mid \Delta$. Write $\Delta=-p^{2 k} m$. (In particular, $p \mid m$.) Define

$$
\delta= \begin{cases}2, & \Delta \text { is odd }, \\ 1, & \Delta \text { is even } .\end{cases}
$$

Assume that $m \geq(4 / 9) p^{2 k}$. Then $\min \left\{p^{2 k},\left(m+\left(p^{k}-\delta\right)^{2}\right) / 4\right\}$ is suitable for $\Delta$.

Proof. Setting

$A_{0}=\frac{p^{2 k}+2 \delta p^{k}-3 \delta^{2}}{3}, \quad A_{1}=3 p^{2 k}-2 \delta p^{k}-\delta^{2}, \quad A_{2}=3 p^{2 k}+2 \delta p^{k}-\delta^{2}$,

a routine verification shows that

$$
\begin{array}{rlrl}
\left(p^{2 k}, p^{2 k}-\delta p^{k}, \frac{m+\left(p^{k}-\delta\right)^{2}}{4}\right) & \in T_{\Delta} & & \text { when } m \geq A_{2}, \quad(3.1) \\
\left(\frac{m+\left(p^{k}-\delta\right)^{2}}{4}, p^{2 k}-\delta p^{k}, p^{2 k}\right) & \in T_{\Delta} & & \text { when } A_{1} \leq m \leq A_{2}, \\
\left(\frac{m+\left(p^{k}-\delta\right)^{2}}{4}, \frac{\left|m-p^{2 k}+\delta^{2}\right|}{2}, \frac{m+\left(p^{k}+\delta\right)^{2}}{4}\right) & \in T_{\Delta} & & \text { when } A_{0} \leq m \leq A_{1}, \\
\frac{m+\left(p^{k}-\delta\right)^{2}}{4} \leq p^{2 k} & \Longleftrightarrow m \leq A_{2} .
\end{array}
$$

The only part of the verification which is not completely trivial is coprimality of the entries of the triple in (3.3). To see this, just note that the difference of the third and the first entry is $\delta p^{k}$. This already proves coprimarity in the case of even $\Delta$, when $\delta=1$, because none of the entries is divisible by $p$. And if $\Delta$ is odd, in which case $\delta=2$, we simply note that the middle entry is odd, because $m \equiv 3 \bmod 4$ (and this is because both $\Delta$ and $p^{2 k}$ are $\left.1 \bmod 4\right)$.

Thus, we have proved that $\min \left\{p^{2 k},\left(m+\left(p^{k}-\delta\right)^{2}\right) / 4\right\}$ is suitable for $\Delta$ when $m \geq A_{0}$. It remains to note that $A_{0} \leq(4 / 9) p^{2 k}$. Indeed, for $x \geq 1$ the function $x \mapsto\left(x^{2}+2 \delta x-3 \delta^{2}\right) / 3 x^{2}$ admits global maximum at $x=3 \delta$, and this maximum is equal to $4 / 9$. 
Proposition 3.4. Let $a$ be a prime number or $a=4$. If $|\Delta| \geq 1000$ then $\Delta$ admits a suitable integer distinct from 1 and $a$.

Proof. There can exist at most 2 integers $b$ satisfying

$$
-a<b \leq a, \quad b^{2} \equiv \Delta \bmod 4 a .
$$

Therefore if the only suitable integers for $\Delta$ are 1 and $a$ then the set $T_{\Delta}$ consists of at most 3 elements. Hence $h(\Delta) \leq 3$. This contradicts the assumption $|\Delta| \geq 1000$, because the largest discriminant with class number 3 is -907 , see Subsection 6.2.

\section{Roots of trinomials and the principal inequal- ity}

In this section we establish some elementary metrical properties of roots of trinomials, both in the complex and $p$-adic setting. The complex result, applied to singular moduli, will yield that non-dominant singular moduli of trinomial discriminant are very close to each other in absolute value. We call this the "principal inequality"; it will indeed be of crucial importance for the rest of the article.

Everything is based on the following property: if $x_{0}, x_{1}, x_{2}$ are roots of a trinomial $t^{m}+A t^{n}+B$ then

$$
\left|\begin{array}{lll}
x_{0}^{m} & x_{0}^{n} & 1 \\
x_{1}^{m} & x_{1}^{n} & 1 \\
x_{2}^{m} & x_{2}^{n} & 1
\end{array}\right|=0 .
$$

\subsection{The complex case}

We start from the following observation.

Proposition 4.1. Let $x_{0}, x_{1}, x_{2} \in \mathbb{C}$ be roots of a trinomial $t^{m}+A t^{n}+B \in \mathbb{C}[t]$. Assume that $\left|x_{0}\right| \geq\left|x_{1}\right| \geq\left|x_{2}\right|$. Then

$$
\left|1-\left(x_{2} / x_{1}\right)^{n}\right| \leq 2\left|x_{1} / x_{0}\right|^{m-n}+2\left|x_{1} / x_{0}\right|^{m}
$$

and

$$
1-\left|x_{2} / x_{1}\right| \leq 2\left|x_{1} / x_{0}\right|+2\left|x_{1} / x_{0}\right|^{3} .
$$

While (4.3) is weaker than (4.2), it has the advantage that $m$ and $n$ are not involved. Hence it may be used to check whether given numbers are roots of some trinomial. This will be used in Section 6 .

Proof. We may assume that $\left|x_{0}\right|>\left|x_{1}\right|$ and $x_{1} \neq x_{2}$, otherwise both results are trivial; in particular, $m \geq 3$. Expanding the determinant in (4.1), we obtain

$$
\begin{aligned}
\left|x_{0}\right|^{m}\left|x_{1}^{n}-x_{2}^{n}\right| & \leq\left|x_{0}\right|^{n}\left(\left|x_{1}\right|^{m}+\left|x_{2}\right|^{m}\right)+\left|x_{1}\right|^{m}\left|x_{2}\right|^{n}+\left|x_{1}\right|^{n}\left|x_{2}\right|^{m} \\
& \leq 2\left|x_{0}\right|^{n}\left|x_{1}\right|^{m}+2\left|x_{1}\right|^{m+n} .
\end{aligned}
$$

Now (4.2) follows dividing by $\left|x_{0}\right|^{m}\left|x_{1}\right|^{n}$, and (4.3) is immediate from (4.2). 
Recall that we call $\Delta$ a trinomial discriminant of signature $(m, n)$ if $h(\Delta) \geq 3$ and the singular moduli of discriminant $\Delta$ are roots of a trinomial of the form $t^{m}+A t^{n}+B$ with rational coefficients.

Corollary 4.2 (The "principal inequality"). Let $x_{1}$ and $x_{2}$ be non-dominant singular moduli of trinomial discriminant $\Delta$ of signature $(m, n)$. Assume that $|\Delta| \geq 1000$ and $\left|x_{1}\right| \geq\left|x_{2}\right|$. Then

$$
\left|1-\left(x_{2} / x_{1}\right)^{n}\right| \leq e^{(m-n)\left(-\pi|\Delta|^{1 / 2}+\log \left|x_{1}\right|+10^{-20}\right)+\log 2} .
$$

In particular, we have the inequalities

$$
\begin{aligned}
& 1-\left|x_{2} / x_{1}\right| \leq e^{(m-n)\left(-\pi|\Delta|^{1 / 2}+\log \left|x_{1}\right|+10^{-20}\right)+\log 2}, \\
& 1-\left|x_{2} / x_{1}\right| \leq e^{-\pi|\Delta|^{1 / 2}+\log \left|x_{1}\right|+0.7}, \\
& 1-\left|x_{2} / x_{1}\right| \leq e^{-\pi|\Delta|^{1 / 2} / 2+0.7} .
\end{aligned}
$$

Proof. Let $x_{0}$ be the dominant singular modulus of discriminant $\Delta$; in particular, $\left|x_{0}\right|>\left|x_{1}\right| \geq\left|x_{2}\right|$. Since $|\Delta| \geq 1000$, we have

$$
\left|\frac{x_{1}}{x_{0}}\right|<\frac{e^{\pi|\Delta|^{1 / 2} / 2}+2079}{e^{\pi|\Delta|^{1 / 2}}-2079}<10^{-21}, \quad\left|x_{0}\right|>e^{\pi|\Delta|^{1 / 2}}-2079>e^{\pi|\Delta|^{1 / 2}-10^{-30}} .
$$

Substituting this to (4.2), we obtain

$$
\begin{aligned}
\left|1-\left(\frac{x_{2}}{x_{1}}\right)^{n}\right| & \leq 2\left|\frac{x_{1}}{x_{0}}\right|^{m-n}+2 \cdot\left(10^{-21}\right)^{n}\left|\frac{x_{1}}{x_{0}}\right|^{m-n} \\
& \leq 2\left(1+10^{-21}\right) e^{(m-n)\left(-\pi|\Delta|^{1 / 2}+\log \left|x_{1}\right|+10^{-30}\right)} \\
& \leq e^{(m-n)\left(-\pi|\Delta|^{1 / 2}+\log \left|x_{1}\right|+10^{-20}\right)+\log 2},
\end{aligned}
$$

which proves (4.4).

Inequality (4.5) follows from (4.4) and both (4.6) and (4.7) follow from (4.5), because $m-n \geq 1$ and

$$
\log \left|x_{1}\right|<\log \left(e^{\pi|\Delta|^{1 / 2} / 2}+2079\right)<\pi|\Delta|^{1 / 2} / 2+10^{-17}
$$

when $|\Delta| \geq 1000$.

\subsection{The non-archimedean case}

In this subsection $K$ is a field of characteristic 0 complete with respect to a non-archimedean absolute value $|\cdot|$. The results of this subsection will be used only in Subsection 6.2 and Section 12

Proposition 4.3. 1. The roots of a trinomial $f(t)=t^{m}+A t^{n}+B \in K[t]$ may have at most 2 distinct absolute values. In other words, the set $\{|x|: x \in K, f(x)=0\}$ consists of at most 2 elements. 
2. Assume now this set has exactly 2 distinct elements $a$ and $b$, with $a>b$. Then for any roots $x_{1}, x_{2}$ with $\left|x_{1}\right|=\left|x_{2}\right|=b$ we have

$$
\left|1-\left(x_{2} / x_{1}\right)^{n}\right| \leq(b / a)^{m-n}
$$

and for any roots $x_{1}, x_{2}$ with $\left|x_{1}\right|=\left|x_{2}\right|=a$ we have

$$
\left|1-\left(x_{2} / x_{1}\right)^{m-n}\right| \leq(b / a)^{n}
$$

Proof. If $x_{0}, x_{1}, x_{2}$ are roots with $\left|x_{0}\right|>\left|x_{1}\right|>\left|x_{2}\right|$ then the determinant in (4.1) has the term $x_{0}^{m} x_{1}^{n}$ which has a strictly larger absolute value than the other 5 terms. Hence the determinant cannot vanish. This proves item 1

Now let $x_{0}, x_{1}, x_{2}$ be roots with $\left|x_{0}\right|=a>\left|x_{1}\right|=\left|x_{2}\right|=b$. Again expanding the determinant, we obtain $a^{m}\left|x_{1}^{n}-x_{2}^{n}\right| \leq a^{n} b^{m}$, which proves (4.8).

Finally, let $x_{0}, x_{1}, x_{2}$ be roots with $\left|x_{0}\right|=b<\left|x_{1}\right|=\left|x_{2}\right|=a$. Then the trinomial $t^{m}+B^{-1} A t^{m-n}+B^{-1}$ has roots $x_{0}^{-1}, x_{1}^{-1}, x_{2}^{-1}$ satisfying

$$
\left|x_{0}^{-1}\right|=b^{-1}>\left|x_{1}^{-1}\right|=\left|x_{2}^{-1}\right|=a^{-1} .
$$

Applying (4.8) in this this set-up, we obtain $\left|1-\left(x_{1}^{-1} / x_{2}^{-1}\right)^{m-n}\right| \leq\left(a^{-1} / b^{-1}\right)^{n}$, which is (4.9).

It turns out that a trinomial having roots of 2 distinct absolute values must have, in the algebraic closure $\bar{K}$, exactly $n$ "small" roots and $m-n$ "big" roots.

Proposition 4.4. In the set-up of item 0 of Proposition 4.3, the trinomial $f(t)$ has exactly $n$ roots $x \in \bar{K}$ with $|x|=b$ and exactly $m-n$ roots $x \in \bar{K}$ with $|x|=a$ (both counted with multiplicities).

Proof. Denote $x_{1}, \ldots, x_{m}$ the roots of $f(t)$ in $\bar{K}$ counted with multiplicities. Let $k$ be the number of roots of absolute value $a$. The coefficient of $t^{m-k}$ in $f(t)$ is given by

$$
(-1)^{k} \sum_{1 \leq i_{1}<\ldots<i_{k} \leq m} x_{i_{1}} \cdots x_{i_{k}} .
$$

In this sum exactly one term is of absolute value $a^{k}$, while the other terms are of strictly smaller absolute value. Hence the coefficient of $t^{m-k}$ does not vanish, which implies that $m-k=n$.

Remark 4.5. As the anonymous referees suggested, item 1 of Proposition 4.3 . and Proposition 4.4 can be proved using the "Newton polygons", as in Section 6.3 of [10]. While our proof of Proposition 4.4 does not use Newton polygons, the present simple argument was inspired by the referees' comments. Our initial statement of Proposition 4.4 was weaker, and the proof was long and ugly. 


\section{Suitable integers for trinomial discriminants}

In this section $\Delta$ denotes a trinomial discriminant unless the contrary is stated explicitly. The following property is crucial.

Proposition 5.1. Let $\Delta$ be a trinomial discriminant admitting at least 2 distinct suitable integers other than 1 . Let $a>1$ be suitable for $\Delta$. Then we have $a>3|\Delta|^{1 / 2} / \log |\Delta|$ if $|\Delta| \geq 10^{5}$, and $a>4|\Delta|^{1 / 2} / \log |\Delta|$ if $|\Delta| \geq 10^{10}$.

(It follows from the proof that, assuming $|\Delta|$ large enough, 4 can be replaced by any $c<2 \pi$.)

Here are some immediate consequences.

Corollary 5.2. Let $\Delta$ be trinomial and $p$ a prime number such that $(\Delta / p)=1$. Then $p>3|\Delta|^{1 / 2} / \log |\Delta|$ if $|\Delta| \geq 10^{5}$ and $p>4|\Delta|^{1 / 2} / \log |\Delta|$ if $|\Delta| \geq 10^{10}$.

Proof. Assume that $|\Delta| \geq 10^{5}$ and $p \leq 3|\Delta|^{1 / 2} / \log |\Delta|$. Then $p \leq|\Delta|^{1 / 2} / 2$, which implies that $p$ is suitable for $\Delta$ by item 3 of Proposition 3.1. Proposition 3.4 implies now that $\Delta$ admits a suitable integer other than 1 and $p$. Hence $p>3|\Delta|^{1 / 2} / \log |\Delta|$ by Proposition 5.1, a contradiction. The case $|\Delta| \geq 10^{10}$ is treated similarly.

Corollary 5.3. Let $\Delta$ be trinomial, $|\Delta| \geq 10^{5}$, and $a>1$ suitable for $\Delta$. Assume that $\operatorname{gcd}(a, \Delta)=1$. Then $a$ is a prime number, and $a>3|\Delta|^{1 / 2} / \log |\Delta|$. Moreover, if $|\Delta| \geq 10^{10}$ then $a>4|\Delta|^{1 / 2} / \log |\Delta|$.

Proof. If $a$ is composite then it has a prime divisor $p$ satisfying $p \leq a^{1 / 2}$. This $p$ is also suitable for $\Delta$ by item 2 of Proposition 3.1, and Proposition 5.1 implies that $p>3|\Delta|^{1 / 2} / \log |\Delta|$. Hence

$$
|\Delta| \geq 3 a^{2} \geq 3 p^{4} \geq 243|\Delta|^{2} /(\log \mid \Delta)^{4},
$$

or $(\log |\Delta|)^{4} \geq 243|\Delta|$, which is clearly impossible when $|\Delta| \geq 10^{5}$. Thus, $a$ is prime, and we complete the proof using Corollary 5.2 .

Before proving Proposition [5.1, we obtain the following preliminary statement.

Proposition 5.4. Let $\Delta$ be a trinomial discriminant, $|\Delta| \geq 10^{5}$. Then it admits at most one suitable a satisfying $1<a \leq 3.4|\Delta|^{1 / 2} / \log |\Delta|$. If $|\Delta| \geq 10^{10}$ then 3.4 can be replaced by 4.5 .

Proof. Assume that $\Delta$ admits suitable $a_{1}$ and $a_{2}$ satisfying

$$
1<a_{1}<a_{2} \leq \kappa \frac{|\Delta|^{1 / 2}}{\log |\Delta|}
$$


with $\kappa$ to be specified later. Let $\left(a_{1}, b_{1}, c_{1}\right),\left(a_{2}, b_{2}, c_{2}\right) \in T_{\Delta}$ be triples where our $a_{1}, a_{2}$ occur, and $x_{1}, x_{2}$ the corresponding singular moduli. We have

$$
\begin{aligned}
\left|x_{1}\right|-\left|x_{2}\right| & \geq e^{\pi|\Delta|^{1 / 2} / a_{1}}-e^{\pi|\Delta|^{1 / 2} / a_{2}}-4158 \\
& =e^{\pi|\Delta|^{1 / 2} / a_{2}}\left(e^{\pi|\Delta|^{1 / 2}\left(1 / a_{1}-1 / a_{2}\right)}-1\right)-4158 \\
& \geq e^{\pi \log |\Delta| / \kappa} \cdot \pi|\Delta|^{1 / 2}\left(\frac{1}{a_{1}}-\frac{1}{a_{2}}\right)-4158 \\
& \geq \frac{\pi|\Delta|^{\pi / \kappa+1 / 2}}{a_{1} a_{2}}-4158 \\
& \geq \frac{\pi}{\kappa^{2}}|\Delta|^{\pi / \kappa-1 / 2}(\log |\Delta|)^{2}-4158 .
\end{aligned}
$$

A calculation shows that

$$
\frac{\pi}{\kappa^{2}}|\Delta|^{\pi / \kappa-1 / 2}(\log |\Delta|)^{2}-4158 \geq 500
$$

when $\kappa=3.4$ and $|\Delta| \geq 10^{5}$, or when $\kappa=4.5$ and $|\Delta| \geq 10^{10}$. (In fact, in the latter case 500 can be replaced by 3600.) Hence $1-\left|x_{2} / x_{1}\right| \geq 500\left|x_{1}\right|^{-1}$. Comparing this with the "principal inequality" (4.7), we obtain

$$
\left|x_{1}\right| \geq 500 e^{\pi|\Delta|^{1 / 2} / 2-0.7},
$$

which is impossible because $\left|x_{1}\right| \leq e^{\pi|\Delta|^{1 / 2} / 2}+2079$.

Proof of Proposition 5.1. Let 1 and $a_{1}>1$ be the smallest suitable integers for $\Delta$. By the assumption, $\Delta$ admits a suitable integer $a_{2}>a_{1}$. Proposition 5.4 implies that $a_{2} \geq 3.4|\Delta|^{1 / 2} / \log |\Delta|$, where 3.4 can be replaced by 4.5 if $|\Delta| \geq 10^{10}$.

Now assume that $a_{1} \leq 3|\Delta|^{1 / 2} / \log |\Delta|$. We will see that this leads to a contradiction. We again let $x_{1}$ and $x_{2}$ be singular moduli for $a_{1}$ and $a_{2}$. Then

$$
\frac{\left|x_{2}\right|}{\left|x_{1}\right|} \leq \frac{\pi|\Delta|^{\pi / 3.4}+2079}{\pi|\Delta|^{\pi / 3}-2079} \leq \frac{|\Delta|^{-0.12}+2079 \pi^{-1}|\Delta|^{-\pi / 3}}{1-2079 \pi^{-1}|\Delta|^{-\pi / 3}} .
$$

When $|\Delta| \geq 10^{5}$, the right-hand side of (5.1) does not exceed 0.3. Then

$$
1-\left|x_{2} / x_{1}\right| \geq 0.7
$$

which clearly contradicts (4.7).

Now assume that $|\Delta| \geq 10^{10}$ and $a_{1} \leq 4|\Delta|^{1 / 2} / \log |\Delta|$. Then instead of (5.1) we have

$$
\frac{\left|x_{2}\right|}{\left|x_{1}\right|} \leq \frac{\pi|\Delta|^{\pi / 4.5}+2079}{\pi|\Delta|^{\pi / 4}-2079}=\frac{|\Delta|^{-\pi / 36}+2079 \pi^{-1}|\Delta|^{-\pi / 4}}{1-2079 \pi^{-1}|\Delta|^{-\pi / 4}} .
$$

The right-hand side is again bounded by 0.3 , and we complete the proof in the same way. 
Another important property of suitable integers is that they are of the same order of magnitude.

Proposition 5.5. Let $a_{1}, a_{2} \neq 1$ be suitable integers for a trinomial discriminant $\Delta$. Then $a_{2}<5 a_{1}$.

Proof. We assume that $a_{2} \geq 5 a_{1}$ and will obtain a contradiction arguing as in the proof of Proposition 5.4. Let $x_{1}$ and $x_{2}$ be singular moduli corresponding to $a_{1}$ and $a_{2}$. Then

$$
\left|x_{1}\right|-\left|x_{2}\right| \geq e^{\pi|\Delta|^{1 / 2} / a_{2}} \cdot \pi|\Delta|^{1 / 2}\left(\frac{1}{a_{1}}-\frac{1}{a_{2}}\right)-4158 .
$$

Using $5 a_{1} \leq a_{2} \leq|\Delta / 3|^{1 / 2}$, we obtain

$$
\begin{aligned}
\left|x_{1}\right|-\left|x_{2}\right| & \geq e^{\pi|\Delta|^{1 / 2} / a_{2}} \cdot \pi|\Delta|^{1 / 2} \cdot \frac{4}{a_{2}}-4158 \\
& \geq e^{\pi \sqrt{3}} \cdot 4 \pi \sqrt{3}-4158 \\
& >800
\end{aligned}
$$

Hence $1-\left|x_{2} / x_{1}\right|>800 /\left|x_{1}\right|$, which leads to a contradiction exactly as in the proof of Proposition [5.4.

\section{Small discriminants}

Recall that we call a discriminant $\Delta$ trinomial if $h(\Delta) \geq 3$ and singular moduli of this discriminant are roots of a trinomial with rational coefficients.

In this section we show that trinomial discriminants must be odd and not too small.

Theorem 6.1. Let $\Delta$ be trinomial discriminant. Then $\Delta$ is odd and satisfies $|\Delta|>10^{11}$.

This theorem is an immediate consequence of Theorem 6.2. Proposition 6.3 and Theorem 6.6 proved below.

Most of the arguments of this section are computer-assisted. We use packages PARI 26] and SAGE [27]. The reader may consulthttps://github.com/yuribilu/trinomials to view our PARI scripts. All computation were performed on a personal computer with $2.70 \mathrm{GHz}$ processor and 16.0 GB RAM.

\subsection{Small discriminants with class number larger than 3}

In this subsection we prove the following.

Theorem 6.2. There are no trinomial discriminants $\Delta$ with $|\Delta| \leq 10^{11}$ and $h(\Delta)>3$.

First of all, we show that sufficiently large trinomial discriminants must be odd. 
Proposition 6.3. Let $\Delta$ be a trinomial discriminant with $|\Delta| \geq 10^{5}$. Then $\Delta$ is odd.

Proof. If $\Delta$ is even but $\Delta \not \equiv 4 \bmod 32$ then, according to item 4 of Proposition [3.1 there is $a \in\{2,4\}$ suitable for $\Delta$. Proposition 3.4 implies that $\Delta$ admits a suitable integer other than 1 and $a$, and Proposition 5.1 implies that $4 \geq a \geq 3|\Delta|^{1 / 2} /(\log |\Delta|)$, which is impossible when $|\Delta| \geq 10^{5}$.

If $\Delta \equiv 4 \bmod 32$ then item 5 of Proposition 3.1 tells us that 8 and 16 are suitable for $\Delta$. Hence $8 \geq 3|\Delta|^{1 / 2} /(\log |\Delta|)$ by Proposition 5.1 which is again impossible when $|\Delta| \geq 10^{5}$.

Next, we dispose of the discriminants in the range $|\Delta| \leq 10^{5}$.

Proposition 6.4. There are no trinomial discriminants $\Delta$ with $|\Delta| \leq 10^{5}$ and $h(\Delta)>3$.

Proof. The proof is by a PARI script. For every such $\Delta$ our script finds singular moduli $x_{0}, x_{1}, x_{2}$ of discriminant $\Delta$ such that inequality (4.3) does not hold. More precisely, for every $\Delta$ in the range $|\Delta| \leq 10^{5}$, except $\Delta=-1467$, our script finds $x_{0}, x_{1}, x_{2}$ satisfying

$$
1-\left|x_{2} / x_{1}\right|>2\left|x_{1} / x_{0}\right|+2\left|x_{1} / x_{0}\right|^{3}+0.15 .
$$

For the exceptional $\Delta=-1467$ one has the same inequality, but with 0.001 instead of 0.15 .

The total running time was less than 6 minutes.

Unfortunately, this method fails for discriminants with class number 3. Each of those admits one real singular modulus $x_{0}$ (the dominant one) and two complex conjugate singular moduli $x_{1}, x_{2}=\bar{x}_{1}$; for them inequality (4.3) is trivially true. In Subsection 6.2 we use a totally different method to show that discriminants with $h=3$ cannot be trinomial.

To dismiss larger discriminants, we show that they admit a small prime $p$ with $(\Delta / p)=1$.

Proposition 6.5. 1. Every odd discriminant $\Delta$ with $|\Delta| \leq 10^{11}$ admits a prime $p \leq 163$ such that $(\Delta / p)=1$.

2. Every odd discriminant $\Delta$ with $|\Delta| \leq 10^{6}$ admits a prime $p \leq 79$ such that $(\Delta / p)=1$.

Proof. We use again a PARI script. It works in 3 steps. In what follows $X$ is a (large) positive number and $p_{0}$ is the largest prime number such that

$$
N_{0}=8 \prod_{3 \leq p \leq p_{0}} p<X,
$$

the product being over primes $p$ in the indicated range. Let also $p_{1}, p_{2}$ be the first two primes larger than $p_{0}$. We have $p_{0}=29, p_{1}=31, p_{2}=37$ for $X=10^{11}$ and $p_{0}=13, p_{1}=17, p_{2}=19$ for $X=10^{6}$. 
Building the list of residues In the first step we use successively the Chinese Remainder Theorem to generate the list of residues $n \bmod N_{0}$ such that $n \equiv 5 \bmod 8$ and $(n / p) \neq 1$ for every odd prime $p \leq p_{0}$. There are

$$
\prod_{3 \leq p \leq p_{0}} \frac{p+1}{2}
$$

such residues altogether, which gives 16329600 residues for $X=10^{11}$ and 1008 residues for $X=10^{6}$.

Building the list of discriminants For every residue class $n \bmod N_{0}$ from the previous list, and every residue class $m \bmod p_{1}$ with $\left(m / p_{1}\right) \neq 1$ we find the smallest negative number $\Delta$ belonging to both, and we include this $\Delta$ in the list only if $|\Delta| \leq X$. We obtain the full list of odd discriminants $\Delta$ with the properties $|\Delta| \leq X$ and $(\Delta / p) \neq 1$ for all $p \leq p_{1}$ (including $\left.p=2\right)$. We end up with 32567861 discriminants for $X=10^{11}$ and with 4450 discriminants for $X=10^{6}$.

Sieving Now we sieve our list modulo every prime $p \geq p_{2}$, by deleting from the list the discriminants $\Delta$ with $(\Delta / p)=1$. The list was emptied after $p=163$ for $X=10^{11}$ and after $p=79$ for $X=10^{6}$.

The bottleneck steps are building the list of discriminants and sieving modulo $p_{2}$ : they require most of processor time and memory. The total running time was less than 5 minutes for $X=10^{11}$ and less than 0.1 second for $X=10^{6}$.

Now we are ready to prove Theorem 6.2 .

Proof of Theorem 6.2. The range $|\Delta| \leq 10^{5}$ is Proposition 6.4 Now assume that $\Delta$ is trinomial in the range $10^{5} \leq|\Delta| \leq 10^{11}$. Then $\Delta$ is odd by Proposition 6.3. If $10^{6} \leq|\Delta| \leq 10^{11}$ then $(\Delta / p)=1$ for some prime $p \leq 163$. Corollary 5.2 implies that $163>3|\Delta|^{1 / 2} / \log |\Delta|$, which is impossible when $|\Delta| \geq 10^{6}$.

Similarly, if $10^{5} \leq|\Delta| \leq 10^{6}$ then $(\Delta / p)=1$ for some prime $p \leq 79$. Hence $79>3|\Delta|^{1 / 2} / \log |\Delta|$, which is impossible when $|\Delta| \geq 10^{5}$.

\subsection{Discriminants with class number 3}

In this subsection we prove the following theorem.

Theorem 6.6. There are no trinomial discriminants with class number 3 .

There are 25 discriminants with class number 3: the full list of them, found by SAGE command cm_orders, is the top row of Table 1 below. As we have seen, they cannot be dismissed using the method of Proposition 6.4. Instead, we use a version of the argument from [19. 
We start by some general discussion. Assume that we are in the following situation: $F(t)=t^{3}+a t^{2}+b t+c \in \mathbb{Z}[t]$ is a $\mathbb{Q}$-irreducible polynomial with splitting field $L$ of degree 6 , and $p$ is a prime number such that

$$
p \mid c \text {, but } p \nmid b \text {. }
$$

Assume further that

$$
p \text { is unramified in } L \text { and inert in the quadratic subfield of } L \text {. }
$$

(The latter assumption can be suppressed, but it holds in all cases that interest us, and many arguments below simplify when it is imposed.)

Let $x_{0}, x_{1}, x_{2} \in L$ be the roots of $F$. Since $p \mid c$, every $x_{i}$ must be divisible by a prime ideal above $p$. Since $p \nmid b$, these ideals must be distinct. Hence $p$ splits in $L$ in at least 3 distinct primes. Since $p$ is inert in the quadratic subfield, it splits in exactly 3 primes: $p=\mathfrak{p}_{0} \mathfrak{p}_{1} \mathfrak{p}_{2}$, with $\mathfrak{p}_{i} \mid x_{i}$. Since $p$ is unramified, we have $\nu_{\mathfrak{p}_{i}}\left(x_{i}\right)=\nu_{p}(c)$ for $i=0,1,2$.

Now assume that $F(t)$ divides a trinomial $t^{m}+A t^{n}+B \in \mathbb{Q}[t]$. Then, setting $\theta=x_{2} / x_{1}$, Proposition 4.3 implies that

$$
\nu_{\mathfrak{p}_{0}}\left(1-\theta^{m-n}\right) \geq n \nu_{\mathfrak{p}_{0}}\left(x_{0}\right)=n \nu_{p}(c) .
$$

To make use of this, we need the following classical fact. Some versions of it were known already to Lucas [20] or even earlier, but we prefer to include the proof for the reader's convenience.

Proposition 6.7. Let $p$ be a prime number, $L$ a number field, $\mathfrak{p} \mid p$ a prime of $L$ and $\theta \in L$ a $\mathfrak{p}$-adic unit, but not a root of unity. Assume that $\mathfrak{p}$ is unramified over $p$. Let $r$ be the order of $\theta \bmod \mathfrak{p}$ (the smallest positive integer such that $\left.\theta^{r} \equiv 1 \bmod \mathfrak{p}\right)$. Then for every positive integer $k$ such that $\theta^{k} \equiv 1 \bmod \mathfrak{p}$ we have $r \mid k$. Furthermore, for $p>2$ we have

$$
\nu_{p}(k)=\nu_{\mathfrak{p}}\left(1-\theta^{k}\right)-\nu_{\mathfrak{p}}\left(1-\theta^{r}\right),
$$

and for $p=2$ we have

$$
\nu_{2}(k)= \begin{cases}\nu_{\mathfrak{p}}\left(1-\theta^{k}\right)-\nu_{\mathfrak{p}}\left(1-\theta^{r}\right), & 2 \nmid k, \\ \nu_{\mathfrak{p}}\left(1-\theta^{k}\right)-\nu_{\mathfrak{p}}\left(1-\theta^{2 r}\right)+1, & 2 \mid k .\end{cases}
$$

Proof. We only have to prove (6.4) and (6.5), because $r \mid k$ is obvious. Note also that $r$ divides $\mathcal{N} \mathfrak{p}-1$, the order of the multiplicative group modp; in particular, $p \nmid r$.

Assume first that $p \nmid k$. Then

$$
1-\theta^{k}=\left(1-\theta^{r}\right)\left(1+\theta^{r}+\theta^{2 r}+\cdots+\theta^{k-r}\right) .
$$

Since $\theta^{r} \equiv 1 \bmod \mathfrak{p}$, the second factor is congruent to $k / r$ modulo $\mathfrak{p}$. In particular, it is not divisible by $\mathfrak{p}$. Hence $\nu_{\mathfrak{p}}\left(1-\theta^{k}\right)=\nu_{\mathfrak{p}}\left(1-\theta^{r}\right)$, and both (6.4), (6.5) are true in this case. 
Now assume that $p \mid k$ and $p>2$. Write $\theta^{k / p}=1+\beta$. Since $r \mid k / p$, we have $\nu_{\mathfrak{p}}(\beta) \geq 1$. Hence

$$
\theta^{k}=1+p \beta+\beta^{p}+\left(\text { terms of } \mathfrak{p} \text {-adic valuation }>\nu_{\mathfrak{p}}(p \beta)\right) .
$$

Since $\mathfrak{p}$ is unramified and $p>2$, we have $\nu_{\mathfrak{p}}\left(\beta^{p}\right)>\nu_{\mathfrak{p}}(p \beta)$ as well. Hence

$$
\nu_{\mathfrak{p}}\left(1-\theta^{k}\right)=\nu_{\mathfrak{p}}(p \beta)=1+\nu_{\mathfrak{p}}\left(1-\theta^{k / p}\right),
$$

and (6.4) follows by induction in $\nu_{p}(k)$.

Now let $p=2$. When $2 \| k$ we can prove that $\nu_{\mathfrak{p}}\left(1-\theta^{k}\right)=\nu_{\mathfrak{p}}\left(1-\theta^{2 r}\right)$ in the same way as we proved $\nu_{\mathfrak{p}}\left(1-\theta^{k}\right)=\nu_{\mathfrak{p}}\left(1-\theta^{r}\right)$ when $p \nmid k$. Hence 6.5. is true is this case. Now assume that $4 \mid k$ and write

$$
\theta^{k / 4}=1+\alpha, \quad \theta^{k / 2}=1+\beta .
$$

Since $r \mid k / 4$, we have $\nu_{\mathfrak{p}}(\alpha) \geq 1$ and $\nu_{\mathfrak{p}}(\beta)=\nu_{\mathfrak{p}}\left(2 \alpha+\alpha^{2}\right) \geq 2$. Since $\mathfrak{p}$ is unramified, this implies that $\nu_{2}\left(2 \beta+\beta^{2}\right)=1+\nu_{2}(\beta)$, or, in other words,

$$
\nu_{\mathfrak{p}}\left(1-\theta^{k}\right)=1+\nu_{\mathfrak{p}}\left(1-\theta^{k / 2}\right),
$$

and we complete the proof by induction as before.

Comparing Proposition 6.7 and inequality (6.3), we obtain the following consequence.

Corollary 6.8. Let $F(t)$ be as above and let p satisfy (6.1) and (6.2). Define $\theta$ and $\mathfrak{p}_{0}$ as above. Assume that $F(t)$ divides a trinomial $t^{m}+A t^{n}+B \in \mathbb{Q}[t]$. Then

$$
m-n \geq r_{0} p^{\nu_{p}(c) n-\nu_{0}},
$$

where $r_{0}$ is the order of $\theta=x_{2} / x_{1}$ modulo $\mathfrak{p}_{0}$, and

$$
\nu_{0}= \begin{cases}\nu_{\mathfrak{p}_{0}}\left(1-\theta^{r_{0}}\right), & p>2, \\ \nu_{\mathfrak{p}_{0}}\left(1-\theta^{2 r_{0}}\right)-1, & p=2 .\end{cases}
$$

Proof. We have $\nu_{\mathfrak{p}_{0}}\left(1-\theta^{m-n}\right)>0$ by (6.1) and (6.3). Proposition 6.7 implies that $r_{0} \mid m-n$. Furthermore, for $p>2$ we use (6.1) and (6.4) to obtain

$$
\nu_{p}(m-n)=\nu_{\mathfrak{p}_{0}}\left(1-\theta^{m-n}\right)-\nu_{\mathfrak{p}_{0}}\left(1-\theta^{r_{0}}\right) \geq n \nu_{p}(c)-\nu_{0} .
$$

Thus, both $r_{0}$ and $p^{n \nu_{p}(c)-\nu_{0}}$ divide $m-n$. Since $p \nmid r_{0}$, this proves (6.6) in the case $p>2$.

In the case $p=2$ the same argument gives

$$
\nu_{2}(m-n) \geq n \nu_{2}(c)-\max \left\{\nu_{\mathfrak{p}_{0}}\left(1-\theta^{r_{0}}\right), \nu_{\mathfrak{p}_{0}}\left(1-\theta^{2 r_{0}}\right)-1\right\} .
$$

We have clearly $\nu_{\mathfrak{p}_{0}}\left(1-\theta^{2 r_{0}}\right) \geq \nu_{\mathfrak{p}_{0}}\left(1-\theta^{r_{0}}\right)+1$, which implies that the maximum above is $\nu_{0}$. 
Table 1: Data for Proposition 6.10

\begin{tabular}{c|cccccccccccccc}
$\Delta$ & -23 & -31 & -44 & -59 & -76 & -83 & -92 & -107 & -108 & -124 & -139 & -172 & -211 \\
\hline$p$ & 17 & 23 & 29 & 11 & 53 & 2 & 53 & 17 & 17 & 89 & 23 & 113 & 29 \\
$r_{0}$ & 1 & 1 & 1 & 1 & 18 & 1 & 1 & 1 & 1 & 1 & 1 & 38 & 1 \\
$\nu_{0}$ & 1 & 1 & 1 & 2 & 1 & 2 & 1 & 1 & 1 & 1 & 1 & 1 & 1 \\
$\lambda$ & 54 & 91 & 83 & 193 & 6.4 & 4.6 & 150 & 25 & 34 & 209 & 34 & 6 & 49 \\
$\mu$ & 6.4 & 7.5 & 5.8 & 23 & 0.6 & 0.8 & 7.5 & 2.4 & 2.1 & 8.8 & 2.4 & 0.3 & 2.4 \\
$\Delta$ & -243 & -268 & -283 & -307 & -331 & -379 & -499 & -547 & -643 & -652 & -883 & -907 \\
\hline$p$ & 23 & 197 & 53 & 47 & 59 & 71 & 83 & 101 & 113 & 389 & 113 & 167 \\
$r_{0}$ & 1 & 11 & 18 & 1 & 1 & 1 & 28 & 1 & 19 & 2 & 38 & 56 \\
$\nu_{0}$ & 1 & 1 & 1 & 1 & 2 & 1 & 1 & 1 & 1 & 1 & 1 & 1 \\
$\lambda$ & 35 & 41 & 4 & 65 & 6447 & 138 & 6.2 & 152 & 8.1 & 480 & 5.1 & 4.7 \\
$\mu$ & 1.7 & 1.5 & 0.2 & 3 & 215 & 4.2 & 0.3 & 4.4 & 0.5 & 9.2 & 0.2 & 0.2
\end{tabular}

Remark 6.9. 1. The lower bound (6.6) is quite strong, but to profit from it in practical situations, we must be able to calculate $r_{0}$ and $\nu_{0}$. We do it as follows. Let $F_{k}(t)$ be the monic polynomial of degree 3 whose roots are $x_{0}^{k}, x_{1}^{k}, x_{2}^{k}$, and $\mathcal{D}_{k}$ its discriminant. Then $\nu_{\mathfrak{p}_{0}}\left(1-\theta^{k}\right)=\nu_{p}\left(\mathcal{D}_{k}\right) / 2$, because $\mathcal{N}_{L / \mathbb{Q}}\left(x_{1}^{k}-x_{2}^{k}\right)=\mathcal{D}_{k}$ and $\mathcal{N}_{L / \mathbb{Q}} \mathfrak{p}_{0}=p^{2}$. In particular, $r_{0}$ is the smallest positive $k$ for which $p \mid \mathcal{D}_{k}$ and

$$
\nu_{0}= \begin{cases}\nu_{p}\left(\mathcal{D}_{r_{0}}\right) / 2, & p>2, \\ \nu_{2}\left(\mathcal{D}_{2 r_{0}}\right) / 2-1, & p=2 .\end{cases}
$$

2. Polynomials $F_{k}(t)=t^{3}+a_{k} t^{2}+b_{k} t+c_{k}$ are very easy to calculate consecutively. Indeed,

$$
\begin{aligned}
& F_{0}(t)=t^{3}-3 t^{2}+3 t-1, \quad F_{1}(t)=F(t), \\
& F_{2}(t)=t^{3}+\left(-a^{2}+2 b\right) t^{2}+\left(b^{2}-2 a c\right) t-c^{2},
\end{aligned}
$$

and for general $k$ we have $c_{k}=-(-c)^{k}$,

$$
a_{k}=-x_{0}^{k}-x_{1}^{k}-x_{2}^{k}, \quad b_{k}=\left(-c / x_{0}\right)^{k}+\left(-c / x_{1}\right)^{k}+\left(-c / x_{2}\right)^{k},
$$

which implies the recurrence relations

$$
a_{k+3}=-a a_{k+2}-b a_{k+1}-c a_{k}, \quad b_{k+3}=b b_{k+2}-a c b_{k+1}+c^{2} b_{k} .
$$

Theorem [6.6] is an easy consequence of the following statement.

Proposition 6.10. Let $\Delta$ be a discriminant with class number 3. Assume that $\Delta$ is trinomial of signature $(m, n)$. Then $p^{3 n}<\lambda n+\mu$, where $p, \lambda, \mu$ can be found in Table 1 .

Proof. It is again by a PARI script. Let $H(t)$ be the Hilbert Class Polynomial of $\Delta$ (the monic polynomial whose roots are the singular moduli of discriminant $\Delta$ ). A verification with PARI shows that, for each of the 25 possible $\Delta$, it has one real root (the dominant one) and two complex conjugate roots. 
Let $d$ be the largest positive integer such that $d^{-3} H(d t) \in \mathbb{Z}[t]$. We want to apply Corollary 6.8 to the polynomial

$$
F(t)=d^{-3} H(d t)=t^{3}+a t^{2}+b t+c .
$$

We pick a prime number $p$ such that $p \mid c$, but $p \nmid b$; our script shows the existence of at least one such $p$ in all the 25 cases. If there are several $p$ with this property, we take the largest of them. The prime chosen for each $\Delta$ can be seen in Table 1 As we verified, each of our primes satisfies $(\Delta / p)=-1$, which means that it is unramified in $L$ (the splitting field of $F$ ) and inert in $\mathbb{Q}(\sqrt{\Delta})$, the quadratic subfield of $L$, so we are indeed in the set-up of Corollary 6.8.

We calculate $r_{0}$ and $\nu_{0}$ as defined in Corollary 6.8, using the method outlined in Remark 6.9. Their values are in Table 1 as well. Corollary 6.8 implies that (6.6) holds true. As our script verified, we always have $\nu_{p}(c)=3$. This implies the lower bound

$$
m-n \geq r_{0} p^{3 n-\nu_{0}}
$$

Now let us bound $m-n$ in terms of $n$ from above. Let $x_{0}$ be the real root of $F$ and $x_{1}, x_{2}=\bar{x}_{1}$ the complex conjugate roots. (Our definition of $F$ implies that $d x_{0}, d x_{1}, d x_{2}$ are the singular moduli of discriminant $\Delta$, of which one is real and the other two are complex conjugates.) Set $\theta=x_{2} / x_{1}$. Then we have inequality (4.2):

$$
\left|1-\theta^{n}\right| \leq 2\left|x_{1} / x_{0}\right|^{m-n}+2\left|x_{1} / x_{0}\right|^{m} .
$$

A quick calculation with PARI shows that $\left|x_{1} / x_{0}\right|<0.001$, which implies that

$$
\left|1-\theta^{n}\right|<2.01\left|x_{1} / x_{0}\right|^{m-n} .
$$

On the other hand, we can estimate $\left|1-\theta^{n}\right|$ from below using the classical Liouville inequality: if $\alpha$ is a non-zero complex algebraic number of degree $\delta$, then

$$
|\alpha| \geq \begin{cases}e^{-\delta \mathrm{h}(\alpha)}, & \alpha \in \mathbb{R}, \\ e^{-(\delta / 2) \mathrm{h}(\alpha)}, & \alpha \notin \mathbb{R} .\end{cases}
$$

Here $\mathrm{h}(\cdot)$ is the usual absolute logarithmic height 1 .

Our $\theta$ is an algebraic number of degree 6 , and not a root of unity, because among its $\mathbb{Q}$-conjugates there is $x_{0} / x_{1}$, of absolute value distinct from 1 . Hence we may apply the Liouville inequality to $\alpha=1-\theta^{n}$.

We have clearly $\mathrm{h}(\alpha) \leq n \mathrm{~h}(\theta)+\log 2$. To estimate $\mathrm{h}(\theta)$, note that the $\mathbb{Q}$ conjugates of $\theta$ are the 6 numbers $x_{i} / x_{j}$ with $1 \leq i \neq j \leq 3$. Of them, only $x_{0} / x_{1}$ and $x_{0} / x_{2}$ are greater than 1 in absolute value. Also, $\theta$ is a root of the polynomial

$$
c^{2} \prod_{i \neq j}\left(t-x_{i} / x_{j}\right) \in \mathbb{Z}[t]
$$

\footnotetext{
${ }^{1}$ There is no risk of confusing the height $\mathrm{h}(\cdot)$ and the class number $h(\cdot)$, not only because the former is roman and the latter is italic, but also because the class number notation $h(\cdot)$ does not occur in this subsection, and heights do not occur outside this subsection.
} 
where $c=-x_{0} x_{1} x_{2}=-x_{0}\left|x_{1}\right|^{2}$ is the free term of $F(t)$. Hence

$$
\mathrm{h}(\theta) \leq \frac{1}{6}\left(2 \log \left|x_{0} / x_{1}\right|+2 \log \left|x_{0} x_{1}^{2}\right|\right)=\frac{2}{3} \log \left|x_{0}\right|+\frac{1}{3} \log \left|x_{1}\right| .
$$

It follows that

$$
\left|1-\theta^{n}\right| \geq e^{-(6 / 2)(n \mathrm{~h}(\theta)+\log 2)} \geq e^{-n\left(2 \log \left|x_{0}\right|+\log \left|x_{1}\right|\right)-3 \log 2} .
$$

Together with (6.8) this implies that

$$
m-n<\frac{2 \log \left|x_{0}\right|+\log \left|x_{1}\right|}{\log \left|x_{0} / x_{1}\right|} n+\frac{3 \log 2+\log 2.01}{\log \left|x_{0} / x_{1}\right|} .
$$

Comparing this with the lower bound (6.7), we obtain $p^{3 n}<\lambda n+\mu$ with

$$
\lambda=\frac{2 \log \left|x_{0}\right|+\log \left|x_{1}\right|}{\log \left|x_{0} / x_{1}\right|} \frac{p^{\nu_{0}}}{r_{0}}, \quad \mu=\frac{3 \log 2+\log 2.01}{\log \left|x_{0} / x_{1}\right|} \frac{p^{\nu_{0}}}{r_{0}} .
$$

Upper bounds for $\lambda$ and $\mu$ produced by our script can be found in Table 1 .

The total running time was less than 2 seconds.

Proof of Theorem [6.6. When $\Delta \neq-83,-331$, inequality $p^{3 n}<\lambda n+\mu$, where $p, \lambda, \mu$ are as in Table 1] cannot hold for $n \geq 1$. Indeed, for these 23 values of $\Delta$ we have $p \geq 11, \lambda \leq 480$ and $\mu \leq 23$. Hence we have $11^{3 n}<480 n+23$, which is impossible for $n \geq 1$. For the remaining two values of $\Delta$ we have

$$
\begin{array}{rlrl}
2^{3 n} & <4.6 n+0.8 & & (\Delta=-83), \\
59^{3 n}<6447 n+215 & & (\Delta=-331) .
\end{array}
$$

These inequalities are impossible for $n \geq 1$ as well. The theorem is proved.

\section{Structure of trinomial discriminants}

In this section we prove Theorem 1.4. For convenience, we reproduce the statement here.

Theorem 7.1. A trinomial discriminant must be of the form $-p$ or $-p q$, where $p, q$ are distinct odd prime numbers, $p \not \equiv q \bmod 4$. In particular, a trinomial discriminant is fundamental.

In this section $\Delta$ denotes a trinomial discriminant; in particular, $\Delta$ is odd and $|\Delta| \geq 10^{11}$ by Theorem 6.1 . The proof is split into many steps which correspond to Subsection 7.17 .6 below.

\section{$7.1 \Delta$ may have at most 2 prime divisors}

Assume that $\Delta$ has 3 distinct (odd) prime divisors $p_{1}, p_{2}, p_{3}$. Set $a_{i}=p_{i}^{\nu_{p_{i}}(\Delta)}$ and $a_{i}^{\prime}=\left|\Delta / a_{i}\right|$. We may assume that $3 \leq a_{1}<a_{2}<a_{3}$. We have clearly $a_{i}^{\prime} \geq 3 a_{i}$ for $i=1,2$. Item 6 of Proposition 3.1 implies that both $a_{1}$ and $a_{2}$ are suitable for $\Delta$. Using Proposition 5.1 we obtain $|\Delta|^{1 / 3} \geq a_{1}>4|\Delta|^{1 / 2} / \log |\Delta|$, which is impossible when $|\Delta| \geq 10^{11}$. 


\section{$7.2-\Delta$ is not a square}

Assume that $\Delta=-m^{2}$, with $m \in \mathbb{Z}$. Among the three primes 5,13, 17 there is one, call it $q$, which does not divide $\Delta$. This $q$ must be suitable, because $(\Delta / q)=1$. Corollary 5.2 implies now that $17 \geq q \geq 4|\Delta|^{1 / 2} / \log |\Delta|$, which is impossible when $|\Delta| \geq 10^{11}$.

\subsection{If $\Delta=-p^{k}$ then $\Delta=-p$}

Assume that $\Delta=-p^{k}$, where $p$ is a prime number and $k$ a positive integer. Since $-\Delta$ is not a square, $k$ must be odd. Assume that $k \geq 3$. Let $q$ be an odd prime divisor of $p+4$. Then $(-p / q)=1$, which implies that $(\Delta / q)=1$. In addition to this, $|\Delta| \geq 10^{11}$ implies that $|\Delta| \geq 4\left(|\Delta|^{1 / 3}+4\right)^{2} \geq 4 q^{2}$. Hence $q$ is suitable for $\Delta$, and Corollary 5.3 implies now that

$$
|\Delta|^{1 / 3}+4 \geq q \geq 4|\Delta|^{1 / 2} / \log |\Delta|
$$

which is impossible when $|\Delta| \geq 10^{11}$. Thus, $k=1$ and $\Delta=-p$.

We are left with the case when $\Delta$ has exactly two odd prime divisors $p_{1}$ and $p_{2}$, with $p_{1}<p_{2}$. In the sequel we write

$$
\Delta=-p_{1}^{k_{1}} p_{2}^{k_{2}} .
$$

We want to show that $k_{1}=k_{2}=1$.

\subsection{We have $k_{1} \leq 2$}

Assume that $k_{1} \geq 3$. Let us show first of all that we must have $\left(k_{1}, k_{2}\right)=(3,1)$. Indeed, assume the contrary: $k_{1} \geq 3$ and $k_{1}+k_{2} \geq 5$. Writing $\Delta=-p_{1}^{2} m$, Proposition 3.3 implies that $\min \left\{p_{1}^{2},\left(m+\left(p_{1}-2\right)^{2}\right) / 4\right\}$ is suitable for $\Delta$. However, since $k_{1}+k_{2} \geq 5$, we have $m>4 p_{1}^{2}$, which implies that the minimum is, actually, $p_{1}^{2}$. Thus, $p_{1}^{2}$ is suitable for $\Delta$. Item 6 of Proposition 3.1 implies that one of the three numbers

$$
p_{1}^{k_{1}}, \quad p_{2}^{k_{2}}, \quad\left(p_{1}^{k_{1}}+p_{2}^{k_{2}}\right) / 4
$$

is suitable as well. Since none of these numbers is equal to $p_{1}^{2}$, Proposition 5.1 applies, and we obtain

$$
|\Delta|^{2 / 5} \geq p_{1}^{2} \geq 4|\Delta|^{1 / 2} / \log |\Delta|
$$

which is impossible when $|\Delta| \geq 10^{11}$.

Thus, we have $\left(k_{1}, k_{2}\right)=(3,1)$, that is, $\Delta=-p_{1}^{3} p_{2}$. In this case we have suitable integers

$$
a_{1}=\min \left\{p_{1}^{2},\left(p_{1} p_{2}+\left(p_{1}-2\right)^{2}\right) / 4\right\}, \quad a_{2}=\min \left\{p_{1}^{3}, p_{2},\left(p_{1}^{3}+p_{2}\right) / 4\right\} .
$$


Let us show that $a_{1} \neq a_{2}$. If $a_{1}=a_{2}$ then we must have

$$
a_{1}=\left(p_{1} p_{2}+\left(p_{1}-2\right)^{2}\right) / 4<p_{1}^{2}, \quad a_{2}=\left(p_{1}^{3}+p_{2}\right) / 4 .
$$

It follows that $p_{1}^{3}<4 a_{2}=4 a_{1}<4 p_{1}^{2}$, which implies that $p_{1}=3$. Furthermore, $p_{1} p_{2}<4 a_{1}<4 p_{1}^{2}$, which implies that $p_{2} \leq 11$. Hence

$$
|\Delta|=p_{1}^{3} p_{2} \leq 3^{3} \cdot 11<10^{11},
$$

a contradiction.

Thus, $a_{1} \neq a_{2}$, and Proposition 5.1 applies. If $p_{2}<p_{1}^{2}$ then $a_{2}=p_{2}$, and we have $|\Delta|^{2 / 5} \geq p_{2} \geq 4|\Delta|^{1 / 2} / \log |\Delta|$, which is impossible. Now assume that

$$
p_{2}>p_{1}^{2},
$$

in which case

$$
\frac{p_{1} p_{2}+\left(p_{1}-2\right)^{2}}{4}>\frac{p_{1}}{4} p_{1}^{2} .
$$

When $p_{1} \geq 5$ this implies that $a_{1}=p_{1}^{2}$. When $p_{1}=3$ we have $p_{2} \geq 10^{11} 3^{-3}$ which again implies $a_{1}=p_{1}^{2}$. Thus, $a_{1}=p_{1}^{2}$ in any case. From (7.2) we deduce that $|\Delta|>p_{1}^{5}$, and we end up with (7.1).

This shows that $\left(k_{1}, k_{2}\right) \neq(3,1)$. Hence we proved that $k_{1} \leq 2$.

\subsection{We have $k_{2}=1$}

Assume that $k_{2} \geq 2$. If $k_{1}=1$ then $p_{1}$ is suitable for $\Delta$ by item 6 of Proposition 3.1. Proposition 3.4 implies that there must be a suitable integer distinct from 1 and $p_{1}$. Proposition 5.1 implies now that

$$
|\Delta|^{1 / 3}>p_{1} \geq 4|\Delta|^{1 / 2} / \log |\Delta|
$$

which is impossible when $|\Delta| \geq 10^{11}$.

Thus, $k_{1}=2$. Hence $k_{2} \geq 3$, because $-\Delta$ is not a square.

Item 6 of Proposition 3.1 implies that $p_{1}^{2}$ is suitable. We want to show that there is one more suitable integer, distinct from 1 and $p_{1}^{2}$. If $k_{2} \geq 4$ then an easy application of Proposition 3.3 implies that $p_{2}^{2}$ is suitable, so in the sequel we will assume that $k_{2}=3$, that is, $\Delta=-p_{1}^{2} p_{2}^{3}$. Note that we must have $p_{1} p_{2} \geq 6000$ : otherwise $|\Delta|<\left(p_{1} p_{2}\right)^{3} / 3<10^{11}$, a contradiction.

We consider two cases.

\subsubsection{The case $p_{2} \geq 1.1 p_{1}^{2}$}

Pick $\ell \in\{3,5\}$ to have $\ell \neq p_{1}$. Since $p_{1}^{2} p_{2}>3 p_{1} p_{2}>10^{4}$, the numbers $p_{1}^{2} p_{2}+1$ and $p_{1}^{2} p_{2}+\ell^{2}$ cannot be both powers of 2 . Hence there exists an odd prime $q$ dividing one of them. This $q$ satisfies

$$
q \leq \frac{p_{1}^{2} p_{2}+25}{2}<0.502 p_{1}^{2} p_{2} .
$$


Using the assumption $p_{2} \geq 1.1 p_{1}^{2}$, we obtain

$$
|\Delta|=p_{2}^{2} \cdot p_{1}^{2} p_{2} \geq 1.1\left(p_{1}^{2} p_{2}\right)^{2}>4 q^{2} .
$$

We have clearly $(\Delta / q)=\left(p_{1}^{2} p_{2} / q\right)=1$. Hence $q$ is suitable by item 3 of Proposition 3.1 .

\subsubsection{The case $p_{2} \leq 1.1 p_{1}^{2}$}

Write $\Delta=-p_{2}^{2} m$, where $m=p_{1}^{2} p_{2}>0.9 p_{2}^{2}$. Proposition 3.3 implies that $a=\min \left\{p_{2}^{2},\left(m+\left(p_{2}-2\right)^{2}\right) / 4\right\}$ is suitable for $\Delta$. We have clearly $p_{2}^{2}>p_{1}^{2}$, and also

$$
\frac{m+\left(p_{2}-2\right)^{2}}{4}>\frac{m}{4} \geq \frac{p_{1}^{2} p_{2}}{4}>p_{1}^{2} .
$$

Hence $a>p_{1}^{2}$.

We have showed that in any case $\Delta$ admits a suitable integer distinct from 1 and $p_{1}^{2}$. Hence Proposition 5.1 applies, and we again have (7.1), which leads to a contradiction. Thus, we must have $k_{2}=1$.

\subsection{We have $k_{1}=1$}

The only remaining possibilities are $\Delta=-p_{1}^{2} p_{2}$ and $\Delta=-p_{1} p_{2}$, and we have to dismiss the former. Thus, let us assume that $\Delta=-p_{1}^{2} p_{2}$. Defining

$$
a=\min \left\{p_{1}^{2}, p_{2},\left(p_{1}^{2}+p_{2}\right) / 4\right\},
$$

item 6 of Proposition 3.1 implies that $a$ is suitable for $\Delta$.

Since $-p_{1}^{2} p_{2}$ is a discriminant, $-p_{2}$ is a discriminant as well, and we have two possible cases.

\section{Case 1: The discriminant $-p_{2}$ admits a suitable integer which is not a power of $p_{1}$}

Item 2 of Proposition 3.1 implies that $-p_{2}$ admits a suitable prime $q \neq p_{1}$. Then $\left(-p_{2} / q\right)=1$ and $p_{2} \geq 3 q^{2}$, which implies that $(\Delta / q)=1$ and $|\Delta| \geq 9 p_{2} \geq 27 q^{2}$. Item 3 of Proposition 3.1 implies that $q$ is suitable for $\Delta$ as well.

Now if $p_{2} \geq 3 p_{1}^{4}$ then $a$, defined in (7.3), satisfies

$$
a=p_{1}^{2} \neq q, \quad|\Delta| \geq 3 a^{3} .
$$

Proposition 5.1]implies that $|\Delta / 3|^{1 / 3} \geq a \geq 4|\Delta|^{1 / 2} / \log |\Delta|$, which is impossible when $|\Delta| \geq 10^{11}$.

And if $p_{2} \leq 3 p_{1}^{4}$ then from $p_{2} \geq 3 q^{2}$ we deduce $|\Delta| \geq 3 q^{3}$. Now Corollary 5.3 implies that $|\Delta / 3|^{1 / 3} \geq q \geq 4|\Delta|^{1 / 2} / \log |\Delta|$, which is again impossible. 


\section{Case 2: Every integer suitable for $-p_{2}$ is a power of $p_{1}$}

Item 2 of Proposition 3.1 implies that in this case the list of suitable integers for $-p_{2}$ consists of consecutive powers of $p_{1}$ :

$$
1, p_{1}, p_{1}^{2}, \ldots, p_{1}^{\ell}
$$

The suitable integer 1 occurs in only one triple in $T_{-p_{2}}$, and each of the suitable integers $p_{1}, p_{1}^{2}, \ldots, p_{1}^{\ell}$ occurs in exactly 2 triples. Hence $h\left(-p_{2}\right)=2 \ell+1$.

On the other hand, from $p_{2}^{3} \geq|\Delta| \geq 10^{11}$ we deduce that $p_{2} \geq 4000$, which implies that $h\left(-p_{2}\right)>6$ (the largest fundamental discriminant of class number not exceeding 6 is -3763 , see [29, Table 4 on page 936]). Hence $\ell \geq 3$, or, equivalently, $p_{1}^{3}$ must be suitable for $-p_{2}$. This implies, in particular, that $p_{2} \geq 3 p_{1}^{6}$. Hence $a$, defined in (17.3), is equal to $p_{1}^{2}$. This shows that $p_{1}^{2}$ is suitable for $\Delta$.

We claim that $p_{1}^{3}$ is suitable for $\Delta$ as well. Indeed, since $p_{1}$ is suitable for $-p_{2}$, there exist $b_{1}, c_{1} \in \mathbb{Z}$ such that $b_{1}^{2}-4 p_{1} c_{1}=-p_{2}$ and $0<b_{1}<p_{1}$. Using $p_{2} \geq 3 p_{1}^{6}$ we obtain

$$
c_{1}=\frac{p_{2}+b_{1}^{2}}{4 p_{1}} \geq \frac{3}{4} p_{1}^{5} .
$$

Now a routine verification shows that

$$
\begin{aligned}
\left(p_{1}^{3}, b_{1} p_{1}, c_{1}\right) & \in T_{\Delta} & & \text { when } p_{1} \nmid c_{1}, \\
\left(p_{1}^{3},\left(2 p_{1}-b_{1}\right) p_{1}, c_{1}-b_{1}+p_{1}\right) & \in T_{\Delta} & & \text { when } p_{1} \mid c_{1} .
\end{aligned}
$$

This proves that $p_{1}^{3}$ is suitable for $\Delta$.

Thus, both $p_{1}^{2}$ and $p_{1}^{3}$ are suitable for $\Delta$. Since $p_{2} \geq 3 p_{1}^{6}$, we have $|\Delta| \geq 3 p_{1}^{8}$. Hence $|\Delta / 3|^{1 / 4} \geq p_{1}^{2}$. Proposition 5.1 implies that

$$
|\Delta / 3|^{1 / 4} \geq p_{1}^{2} \geq 4|\Delta|^{1 / 2} / \log |\Delta|,
$$

which is impossible when $|\Delta| \geq 10^{11}$.

This completes the proof of Theorem 7.1 .

\section{Primality of suitable integers}

As before, $\Delta$ denotes a trinomial discriminant unless the contrary is stated explicitly. In particular, $|\Delta|>10^{11}$ by Theorem 6.1, and, according to Theorem 7.1, we have $\Delta=-p$ or $\Delta=-p q$ where $p, q$ are distinct odd prime numbers.

As we have seen in Corollary 5.3 , suitable integers for trinomial discriminants are prime numbers with some rare exceptions. It turns out that there are no exceptions at all.

Proposition 8.1. Let $\Delta$ be a trinomial discriminant and $a>1$ suitable for $\Delta$. Then $a$ is prime and satisfies $a>4|\Delta|^{1 / 2} / \log |\Delta|$. 
Remark 8.2. Since $|\Delta| \geq 10^{11}$, this implies, in particular, that $a>10^{4}$.

Before proving Proposition [8.1 observe that, in the case $\Delta=-p q$, the primes $p, q$ are of the same order of magnitude up to a logarithmic factor.

Proposition 8.3. If $\Delta=-p q$ then

$$
\frac{4|\Delta|^{1 / 2}}{\log |\Delta|}<p, q<\frac{|\Delta|^{1 / 2} \log |\Delta|}{4} .
$$

Proof. It suffices to prove the lower estimate in (8.1); the upper estimate will then follow automatically. Thus, let us assume that $p<q$ and prove that $p>4|\Delta|^{1 / 2} / \log |\Delta|$.

Since $|\Delta|>10^{11}$ we have $4|\Delta|^{1 / 2} / \log |\Delta|<|\Delta|^{1 / 2} / \sqrt{3}$. Hence we may assume that $p<q / 3$, in which case $p$ is suitable for $\Delta$ by item 6 of Proposition 3.1] Proposition 3.4 implies that $\Delta$ has a suitable integer other than 1 and $p$, and Proposition 5.1 implies that $p>4|\Delta|^{1 / 2} / \log |\Delta|$.

Proof of Proposition 8.1. If $\operatorname{gcd}(a, \Delta)=1$ then Corollary 5.3 does the job. In particular, this completes the proof in the case $\Delta=-p$. Now assume that $\Delta=-p q$ with $p<q$ and $\operatorname{gcd}(a, \Delta)>1$. Since $a \leq|\Delta / 3|^{1 / 2}$, the only possibility is $\operatorname{gcd}(a, \Delta)=p$, and we claim that $a=p$.

Indeed, assume that $a>p$, and let $\ell$ be a prime divisor of $a / p$. From $a \leq|\Delta / 3|^{1 / 2}$ and $p>4|\Delta|^{1 / 2} / \log |\Delta|$ we deduce

$$
\ell<0.2 \log |\Delta|<4|\Delta|^{1 / 2} / \log |\Delta|<p .
$$

Hence $\ell$ is coprime with $\Delta$, which implies that it is suitable for $\Delta$, see item 2 of Proposition 3.1. Now Corollary 5.3 implies that $\ell>4|\Delta|^{1 / 2} / \log |\Delta|$, a contradiction.

\section{A conditional result}

In this section we prove Theorem 1.2, Let us reproduce it here for convenience.

Theorem 9.1. Assume GRH. Then a singular modulus of degree at least 3 cannot be a root of a trinomial with rational coefficients. In other words, GRH implies that trinomial discriminants do not exist.

In this section, by the $R H$ we mean the Riemann Hypothesis for the Riemann $\zeta$-function, and by GRH the Generalized Riemann Hypothesis for Dirichlet $L$ functions.

Due to the results of the previous sections, Theorem 9.1 is an easy consequence of the following statement.

Proposition 9.2. Assume GRH. Let $m \geq 10^{10}$ be an integer with $\omega(m) \leq 2$, and $\chi$ a primitive odd real Dirichlet character modulo $m$. Then there exists a prime number $p$ such that $\chi(p)=1$ and

$$
p \leq 4 m^{1 / 2} / \log m \text {. }
$$


Recall that a real character $\chi$ is called odd if $\chi(-1)=-1$. Restricting to odd characters is purely opportunistic here: the same argument, with very insignificant changes, applies to even real primitive characters as well. But we apply estimate (9.1) only to real odd characters, and making this assumption allows us to shorten the proof. The assumption $\omega(m) \leq 2$ is of similar nature: it can be dropped, making the proof a bit more complicated, but this is unnecessary because we will apply (9.1) only to $m$ with at most 2 prime divisors.

Remark 9.3. Unconditionally, the bound $p \ll_{\varepsilon} m^{1 / 4+\varepsilon}$ holds for every $\varepsilon>0$; this is a classical result of Linnik and Vinogradov. Unfortunately, the implied constant in this estimate depends ineffectively on $\varepsilon$, because of ineffectiveness of Siegel's bound for the exception real zero of $L(s, \chi)$. In Section 10 we imitate the Linnik-Vinogradov argument in the form given in [21], but with Siegel's Theorem replaced by Tatuzawa's Theorem [25], obtaining this way an unconditional explicit upper bound for all but one trinomial discriminants.

Proof of Theorem 9.1 (assuming Proposition 9.2). Let $\Delta$ be a trinomial discriminant. Theorem 6.1 implies that $|\Delta| \geq 10^{10}$. We apply Proposition 9.2 with the character $(\Delta / \cdot)$, which is an odd real Dirichlet character $\bmod |\Delta|$. Moreover, it is primitive because $\Delta$ is fundamental, see Theorem 7.1. Note also that $\omega(|\Delta|) \leq 2$, again by Theorem 7.1. We find (assuming GRH) a prime $p$ satisfying $(\Delta / p)=1$ and $p \leq 4|\Delta|^{1 / 2} / \log |\Delta|$, which contradicts Corollary 5.2.

The proof of Proposition9.2 is an adaptation of the argument developed by Lamzouri et al. in [17. Their Theorem 1.4 implies, in our case, the estimate

$$
p \leq \max \left\{10^{9},\left(\log m+\frac{5}{2}(\log \log m)^{2}+6\right)^{2}\right\} .
$$

Of course, it is asymptotically much sharper than (9.1), but (9.2) is not suitable for our purposes because of the term $10^{9}$.

We prove Proposition 9.2 in Subsection 9.2 , after some preparatory work in Subsection 9.1

\subsection{Lemmas from [17]}

In this subsection we recall some technical lemmas proved in 17, and give simplified versions of them. We use the notation of [17] whenever possible; our only major deviation from the set-up of [17] is that we denote the modulus by $m$, while it is usually denoted by $q$ therein.

For $x>1$ and a Dirichlet character $\chi$ define

$$
\begin{array}{ll}
S(x)=\sum_{1 \leq n \leq x} \Lambda(n) \log \frac{x}{n}, & S(x, \chi)=\sum_{1 \leq n \leq x} \chi(n) \Lambda(n) \log \frac{x}{n}, \\
T(x)=\sum_{1 \leq n \leq x} \frac{\Lambda(n)}{n}\left(1-\frac{n}{x}\right), & T(x, \chi)=\sum_{1 \leq n \leq x} \chi(n) \frac{\Lambda(n)}{n}\left(1-\frac{n}{x}\right) .
\end{array}
$$


Here $\Lambda(\cdot)$ is, of course, the von Mangoldt function.

Denote by $\gamma$ the Euler-Mascheroni constant, and define

$$
B=\frac{1}{2} \log (4 \pi)-1-\frac{\gamma}{2}=-0.02309 \ldots
$$

(see equation (2.2) on [17, page 2395]). The following is combination of Lemmas 2.1 and 2.4 from [17.

Lemma 9.4. Assume $R H$. Then for $x>1$ we have

$$
\begin{aligned}
& S(x)=x-(\log 2 \pi) \log x-1+\sum_{k=1}^{\infty} \frac{1-x^{-2 k}}{4 k^{2}}+O_{1}\left(2|B|\left(x^{1 / 2}+1\right)\right), \\
& T(x)=\log x-(1+\gamma)+\frac{\log (2 \pi)}{x}-\sum_{n=1}^{\infty} \frac{x^{-2 n-1}}{2 n(2 n+1)}+O_{1}\left(\frac{2|B|}{x^{1 / 2}}\right),
\end{aligned}
$$

where $B$ is defined in (9.3).

Recall (see Subsection 1.1) that $X=O_{1}(Y)$ means that $|X| \leq Y$.

We will use the following simplified version of this lemma for large $x$.

Lemma 9.5. In the set-up of Lemma 9.4, for $x \geq 100$ we have

$$
S(x)=x-(\log 2 \pi) \log x+O_{1}\left(0.16 x^{1 / 2}\right) \leq 1.02 x .
$$

For $x \geq 10^{4}$ we have

$$
\begin{aligned}
& S(x)=x-(\log 2 \pi) \log x+O_{1}\left(0.06 x^{1 / 2}\right) \\
& T(x)=\log x-(1+\gamma)+O_{1}\left(\frac{0.07}{x^{1 / 2}}\right) \leq \log x-1.576
\end{aligned}
$$

The proof of this lemma is left out, being an easy calculation.

For $x>1$ define

$$
\begin{aligned}
& \widetilde{E}_{1}(x)=\frac{\pi^{2}}{8}-\left(\log 2+\frac{\gamma}{2}\right) \log x-\sum_{k=0}^{\infty} \frac{x^{-2 k-1}}{(2 k+1)^{2}} \\
& E_{1}(x)=-\sum_{k=0}^{\infty} \frac{x^{-2 k-2}}{(2 k+1)(2 k+2)}-\frac{\gamma}{2}\left(1-\frac{1}{x}\right)+\frac{\log 2}{x} .
\end{aligned}
$$

The next lemma combines Lemmas 2.2 and 2.3 from [17, in the special case of odd real characters.

Lemma 9.6. Let $m \geq 3$ be an integer and $\chi$ be a primitive real odd Dirichlet character modulo $m$. Assume GRH. Then for $x>1$ we have

$$
S(x, \chi)=R(\chi)\left(\log x+O_{1}\left(2 x^{1 / 2}+2\right)\right)+\frac{1}{2}\left(\log \frac{m}{\pi}\right) \log x+\widetilde{E}_{1}(x),
$$

where

$$
R(\chi)=\left(1+\frac{1}{x}+O_{1}\left(\frac{2}{x^{1 / 2}}\right)\right)^{-1}\left(\frac{1}{2}\left(1-\frac{1}{x}\right) \log \frac{m}{\pi}-T(x, \chi)+E_{1}(x)\right) .
$$


Note that we denote by $R(\chi)$ the quantity $|\operatorname{Re}(B(\chi))|$ from [17].

We again give a simplified version (of the lower bound only, we do not need the upper bound).

Lemma 9.7. In the set-up of Lemma 9.6 assume that

$$
m \geq 10^{10}, \quad 10^{4} \leq x \leq 0.2 m^{1 / 2} .
$$

Then

$$
S(x, \chi) \geq-2.1 x^{1 / 2}(\log m-4)-(0.53 \log m-1.9) \log x .
$$

Proof. We have $E_{1}(x) \leq-0.288$ and

$$
|T(x, \chi)| \leq T(x) \leq \log x-1.576 \leq \frac{1}{2} \log m-3.185,
$$

see (9.6). Hence

$$
\begin{aligned}
\frac{1}{2}\left(1-\frac{1}{x}\right) \log \frac{m}{\pi}-T(x, \chi)+E_{1}(x) & \leq \log m-\frac{1}{2} \log \pi-3.185-0.288 \\
& \leq \log m-4 \\
R(\chi) & \leq 1.021(\log m-4) .
\end{aligned}
$$

Furthermore, we have $\widetilde{E}_{1}(x) \geq-(\gamma / 2+\log 2) \log x$. Hence

$$
\frac{1}{2}\left(\log \frac{m}{\pi}\right) \log x+\widetilde{E}_{1}(x) \geq\left(\frac{1}{2} \log m-\log (2 \pi)-\frac{\gamma}{2}\right) \log x .
$$

Substituting (9.9) and (9.10) into (9.7), we obtain (9.8).

Finally, the following is (a consequence of) Lemma 3.1 from [17] (which is unconditional, unlike the previous lemmas).

Lemma 9.8. Let $m \geq 3$ be an integer and $x \geq 2$ be a real number. Then

$$
\sum_{\substack{1 \leq n \leq x \\(n, m)>1}} \Lambda(n) \log (x / n) \leq \frac{1}{2} \omega(m)(\log x)^{2} .
$$

\subsection{Proof of Proposition 9.2}

Assume the contrary: $\chi(p) \neq 1$ for every $p \leq 4 m^{1 / 2} / \log m$. Since $\chi$ is a real character, this implies that

$$
-S(x, \chi) \geq S(x)-\sum_{p \leq x^{1 / 2}} \log p \log \left(x / p^{2}\right)-\sum_{\substack{1 \leq n \leq x \\(n, m)>1}} \Lambda(n) \log (x / n) .
$$


where we set $x=4 m^{1 / 2} / \log m$. Since $m \geq 10^{10}$, we have $10^{4} \leq x \leq 0.2 m^{1 / 2}$, which means that we may use estimates (9.5) and (9.8). We may also use (9.4) with $x$ replaced by $x^{1 / 2}$, which gives

$$
\sum_{p \leq x^{1 / 2}} \log p \log \left(x / p^{2}\right) \leq 2 S\left(x^{1 / 2}\right) \leq 2.04 x^{1 / 2} .
$$

Finally, Lemma 9.8 and the assumption $\omega(m) \leq 2$ imply that

$$
\sum_{\substack{1 \leq n \leq x \\(n, m)>1}} \Lambda(n) \log (x / n) \leq(\log x)^{2} \leq \log \left(0.2 m^{1 / 2}\right) \log x \leq(0.5 \log m-1.6) \log x .
$$

Combining all these estimates, we obtain

$$
\begin{aligned}
2.1 x^{1 / 2}(\log m-4)+(0.53 \log m-1.9) \log x \geq & x-(\log 2 \pi) \log x-0.06 x^{1 / 2} \\
& -2.04 x^{1 / 2}-(0.5 \log m-1.6) \log x,
\end{aligned}
$$

which can be re-written as

$$
2.1 x^{1 / 2}(\log m-3)+(1.03 \log m-1.6) \log x \geq x .
$$

When $x \geq 10^{4}$ the left-hand side does not exceed $(2.2 \log m-6) x^{1 / 2}$, which implies the inequality $x^{1 / 2} \leq 2.2 \log m-6$. Substituting $x=4 m^{1 / 2} / \log m$, we obtain

$$
m^{1 / 4} \leq(1.1 \log m-3)(\log m)^{1 / 2} .
$$

This inequality is impossible when $m \geq 10^{10}$.

\section{Bounding all but one trinomial discriminants}

In this section we prove the following theorem.

Theorem 10.1. There exists at most one trinomial discriminant $\Delta$ satisfying $|\Delta| \geq 10^{160}$.

Call a positive integer $m$ coarse 2 if $m$ is either prime or a product of two distinct primes each exceeding $m^{3 / 8} \log m$. We deduce Theorem 10.1 from the following statement.

Theorem 10.2. With at most one exception, every coarse integer $m>10^{160}$ has the following property. Let $\chi$ be a primitive real Dirichlet character $\bmod m$. Then there exists a prime $p \leq 4 m^{1 / 2} / \log m$ such that $\chi(p)=1$.

Proof of Theorem 10.1 (assuming Theorem 10.2). Let $\Delta$ be a trinomial discriminant satisfying $|\Delta| \geq 10^{160}$. Then $m=|\Delta|$ is either prime or product of two distinct primes both exceeding $4 m^{1 / 2} / \log m$, see Proposition 8.3. Since $m \geq 10^{160}$, it must be coarse. Let $\chi(\cdot)$ be the Kronecker $(\Delta / \cdot)$; then, unless $m$ is the exceptional one from Theorem 10.2 there exists $p \leq 4 m^{1 / 2} / \log m$ such that $\chi(p)=1$, contradicting Corollary 5.2 .

\footnotetext{
2 as opposed to smooth
} 
Theorem 10.2 will be proved in Subsection 10.2, after we establish, in Subsection 10.1, an explicit version of the Burgess estimate for coarse moduli.

\subsection{Explicit Burgess for coarse moduli}

Everywhere in this subsection $m$ is a positive integer and $\chi$ a primitive Dirichlet character $\bmod m$. For $M, N \in \mathbb{Z}$ with $N>0$ denote

$$
S(N, M)=\sum_{M<n \leq M+N} \chi(n) .
$$

A classical result of Burgess [8, 9] implies that $|S(M, N)| \ll_{\varepsilon} N^{1 / 2} m^{3 / 16+\varepsilon}$. We need a version of this inequality explicit in all parameters. Such a version is available in the case of prime modulus [6, 15, 28, but we need a slightly more general version of it, for coarse moduli, as defined in the beginning of Section 10 .

Theorem 10.3. Let $m>10^{11}$ be a coarse integer. Then for every $M, N$ as above we have

$$
|S(N, M)|<10 N^{1 / 2} m^{3 / 16}(\log m)^{1 / 2} .
$$

Note that we did not try to optimize the numerical constant 10. Probably, sharper constants are possible, as the work of Booker [6] and Treviño [28] suggests.

Remark 10.4. After this article was submitted, fully explicit versions of the Burgess inequality with arbitrary composite moduli emerged, see [7, Theorem 1.1] or [16. Theorem 1.1 and Corollary 1.2]. However, for the sake of consistency, we prefer to use our Theorem 10.3 .

The following lemma is quite standard, but we did not find a suitable reference.

Lemma 10.5. Assume that $m$ is square-free, and let $f(x) \in \mathbb{Z}[x]$ be a polynomial with the following property: there exists $b \in \mathbb{Z}$ such that

$$
m \mid f(b), \quad \operatorname{gcd}\left(f^{\prime}(b), m\right)=1 .
$$

Then the sum

$$
S_{\chi}(f)=\sum_{x \bmod m} \chi(f(x))
$$

satisfies $\left|S_{\chi}(f)\right| \leq(\mu-1)^{\omega(m)} m^{1 / 2}$, where $\mu$ is the number of distinct roots of $f$ modulo $m$.

Proof. Let $m=p_{1} \cdots p_{k}$ be the prime factorization of $m$ (recall that $m$ is squarefree), and set $m_{i}=m / p_{i}$. Our character $\chi$ has a unique presentation as $\chi_{1} \cdots \chi_{k}$, where each $\chi_{i}$ is a primitive character $\bmod p_{i}$. Then

$$
S_{\chi}(f)=S_{\chi_{1}}\left(f_{1}\right) \cdots S_{\chi_{k}}\left(f_{k}\right)
$$

where $f_{i}(x)=f\left(m_{i} x\right)$; see, for instance, equation (12.21) in [15. Since each $f_{i}$ has a simple root modulo $p_{i}$, the Hasse-Weil bound $\left|S_{\chi_{i}}\left(f_{i}\right)\right| \leq(\mu-1) p_{i}^{1 / 2}$ applies (see, for instance, 24], Theorem $2 \mathrm{C}^{\prime}$ on page 43 ). The result follows. 
Proof of Theorem 10.3. Denote $E(N)=\max \{|S(N, M)|: M \in \mathbb{Z}\}$. We want to prove that

$$
E(N)<10 N^{1 / 2} m^{3 / 16}(\log m)^{1 / 2} .
$$

We follow rather closely the argument from the book of Iwaniec and Kowalski [15. pages 327-329], where we set $r=2$. In particular, we will use induction in $N$.

If $N<100 m^{3 / 8} \log m$ then (10.1) follows from the trivial estimate $E(N) \leq N$, and if $N \geq m^{5 / 8} \log m$ then (10.1) follows from the Pólya-Vinogradov inequality $E(N) \leq 6 m^{1 / 2} \log m$, see [15, Theorem 12.5]. Hence we may assume in the sequel that

$$
100 m^{3 / 8} \log m \leq N<m^{5 / 8} \log m .
$$

We fix positive integers $A, B$, to be specified later, such that

$$
3 \leq A, B<m^{3 / 8} \log m, \quad A B<N .
$$

Since $m$ is coarse,

$$
\operatorname{gcd}(a, m)=1 \quad(1 \leq a \leq \max \{A, B\}) .
$$

For a residue class $x$ mod $m$ denote by $\nu(x)$ the number of presentation of $x$ as $\bar{a} n$, where

$$
1 \leq a \leq A, \quad M<n \leq M+N,
$$

and $\bar{a}$ denote the inverse of $a$ modulo $m$.

Arguing as in [15, page 327], we find

$$
|S(M, N)| \leq V / H+2 E(H)
$$

where

$$
H=A B, \quad V=\sum_{x \bmod m} \nu(x)\left|\sum_{1 \leq b \leq B} \chi(x+b)\right| .
$$

Using Hölder's inequality, we estimate

$$
V \leq V_{1}^{1 / 2} V_{2}^{1 / 4} W^{1 / 4},
$$

where

$$
V_{1}=\sum_{x \bmod m} \nu(x), \quad V_{2}=\sum_{x \bmod m} \nu(x)^{2}, \quad W=\sum_{x \bmod m}\left|\sum_{1 \leq b \leq B} \chi(x+b)\right|^{4} .
$$

We have the following estimates:

$$
V_{1} \leq N A, \quad V_{2} \leq 8 N A\left(\frac{N A}{m}+\log (3 A)\right), \quad W \leq 9 B^{4} m^{1 / 2}+3 B^{2} m .
$$


Now we are going to complete the proof, assuming them. Estimates (10.6) themselves will be proved afterwards.

Set

$$
A=\left\lfloor 0.04 N m^{-1 / 4}\right\rfloor, \quad B=\left\lfloor m^{1 / 4}\right\rfloor .
$$

From the hypotheses $m>10^{11}$ and the inequality (10.2) we deduce that (10.3) indeed holds. Moreover, $H=A B$ satisfies $0.03 N<H \leq 0.04 N$.

Since $H<N$, estimate (10.1) holds true, by induction, with $N$ replaced by $H$ :

$$
E(H)<10 H^{1 / 2} m^{3 / 16}(\log m)^{1 / 2} \leq 2 N^{1 / 2} m^{3 / 16}(\log m)^{1 / 2} .
$$

Now let us estimate $V$. We have clearly

$$
V_{1} \leq N A \leq 0.04 N^{2} m^{-1 / 4}, \quad W \leq 12 m^{3 / 2} .
$$

Furthermore, using (10.2) and $m>10^{11}$, we obtain

$$
\log (3 A) \leq 0.5 \log m, \quad N A / m \leq 0.04(\log m)^{2} .
$$

It follows that $V_{2} \leq 0.5 N A(\log m)^{2}$. We obtain

$$
\begin{aligned}
V & \leq(N A)^{1 / 2}\left(0.5 N A(\log m)^{2}\right)^{1 / 4}\left(12 m^{3 / 2}\right)^{1 / 4} \\
& <0.14 N^{3 / 2} m^{3 / 16}(\log m)^{1 / 4} .
\end{aligned}
$$

Substituting all this in (10.5), we obtain

$$
\begin{aligned}
|S(M, N)| & <\frac{0.14 N^{3 / 2} m^{3 / 16}(\log m)^{1 / 4}}{0.03 N}+2 \cdot 2 N^{1 / 2} m^{3 / 16}(\log m)^{1 / 2} \\
& <9 N^{1 / 2} m^{3 / 16}(\log m)^{1 / 2}
\end{aligned}
$$

as wanted.

We are left with the estimates from (10.6). The estimate $V_{1} \leq N A$ is obvious. The estimate for $V_{2}$ is Lemma 12.7 from [15, page 328]. The only difference is that in [15] $m$ is a prime number (and denoted $p$ ). However, it is only needed therein that every integer between 1 and $A$ is co-prime with $m$, which is true in our case, see (10.4).

Finally, let us prove the estimate for $W$. The proof is very similar to that of [15. Lemma 12.8]. We write

$$
W=\sum_{1 \leq b_{1} \ldots b_{4} \leq B} \sum_{x} \chi\left(\left(x+b_{1}\right)\left(x+b_{2}\right)\right) \bar{\chi}\left(\left(x+b_{3}\right)\left(x+b_{4}\right)\right) .
$$

Note that $b_{i} \neq b_{j}$ implies that $\operatorname{gcd}\left(b_{i}-b_{j}, m\right)=1$, see (10.4). Now if a quadruple $\left(b_{1}, \ldots, b_{4}\right)$ has the property that

$$
\text { some } b_{i} \text { is distinct from all other } b_{j} \text {, }
$$

then

$$
\left|\sum_{x \bmod m} \chi\left(\left(x+b_{1}\right)\left(x+b_{2}\right)\right) \bar{\chi}\left(\left(x+b_{3}\right)\left(x+b_{4}\right)\right)\right| \leq 9 m^{1 / 2}
$$


by Lemma 10.5 applied to the polynomial

$$
f(x)=\left(x+b_{1}\right)\left(x+b_{2}\right)\left(\left(x+b_{3}\right)\left(x+b_{4}\right)\right)^{\varphi(m)-1} .
$$

(Here, the $b$ from Lemma 10.5 is the $b_{i}$ that is distinct from all other $b_{j}$.) And a simple combinatorial argument shows that exactly $3 B^{2}-2 B$ quadruples do not satisfy (10.7). Hence

$$
W \leq 9 m^{1 / 2}\left(B^{4}-3 B^{2}+2 B\right)+m\left(3 B^{2}-2 B\right),
$$

which is slightly sharper than wanted. The theorem is proved.

\subsection{Proof of Theorem 10.2}

Set

$$
\rho(n)=\sum_{d \mid n} \chi(d) .
$$

Note that $\rho(\cdot)$ is a multiplicative function.

The following statement is a version of Proposition 3.1 from Pollack 21].

Proposition 10.6. Let $m$ and $\chi$ be as in Theorem 10.3 (in particular, $m$ is coarse). Then for $x \geq 10^{4} m^{3 / 8} \log m$ we have

$$
\sum_{1 \leq n \leq x} \rho(n)=x L(1, \chi)+O_{1}\left(50 m^{1 / 8}(\log m)^{1 / 3} x^{2 / 3}\right) .
$$

(Recall that $A=O_{1}(B)$ means $|A| \leq B$.)

Proof. Set $\Theta=10 m^{3 / 16}(\log m)^{1 / 2}$, so that (10.1) can be written as

$$
\left|\sum_{M<n \leq M+N} \chi(n)\right| \leq \Theta N^{1 / 2} .
$$

Let $y$ be a real number satisfying $1 \leq y<x$, to be specified later, and set $z=x / y$. Intuitively, one should think of $y$ as "large" (not much smaller than $x$ ) and $z$ "small". As in 21, we use the "Dirichlet hyperbola formula"

$$
\sum_{1 \leq n \leq x} \rho(n)=\sum_{1 \leq d \leq y} \chi(d) \sum_{1 \leq e \leq x / d} 1+\sum_{1 \leq e \leq z} \sum_{1 \leq d \leq x / e} \chi(d)-\sum_{1 \leq d \leq y} \chi(d) \sum_{1 \leq e \leq z} 1 .
$$

Here the first double sum will give the main contribution, while the second and the third double sums will be absorbed in the error term.

Using (10.10), we estimate the last two double sums:

$$
\begin{aligned}
& \left|\sum_{1 \leq e \leq z} \sum_{1 \leq d \leq x / e} \chi(d)\right| \leq \Theta x^{1 / 2} \sum_{1 \leq e \leq z} \frac{1}{e^{1 / 2}} \leq 2 \Theta x^{1 / 2}\left(1+z^{1 / 2}\right) \leq 4 \Theta x^{1 / 2} z^{1 / 2}, \\
& \left|\sum_{1 \leq d \leq y} \chi(d) \sum_{1 \leq e \leq z} 1\right| \leq \Theta y^{1 / 2} z=\Theta x^{1 / 2} z^{1 / 2} .
\end{aligned}
$$


For the first double sum we have the expression

$$
\sum_{1 \leq d \leq y} \chi(d) \sum_{1 \leq e \leq x / d} 1=\sum_{1 \leq d \leq y} \chi(d)\left\lfloor\frac{x}{d}\right\rfloor=x L(1, \chi)+R_{1}+R_{2},
$$

where

$$
R_{1}=\sum_{1 \leq d \leq y} \chi(d)\left(\left\lfloor\frac{x}{d}\right\rfloor-\frac{x}{d}\right), \quad R_{2}=-x \sum_{d>y} \frac{\chi(d)}{d} .
$$

We have clearly $\left|R_{1}\right| \leq y$. To estimate $R_{2}$ we use partial summation. For an integer $k>y$ set $S_{k}=\sum_{y<n \leq k} \chi(n)$. Using (10.10) we estimate

$$
\left|S_{k}\right| \leq \Theta(k-y)^{1 / 2} \leq \Theta k^{1 / 2} .
$$

Hence

$$
\left|R_{2}\right|=x\left|\sum_{k>y} S_{k}\left(\frac{1}{k}-\frac{1}{k+1}\right)\right| \leq \Theta x \sum_{k>y} \frac{1}{k^{1 / 2}(k+1)} \leq 3 \Theta x y^{-1 / 2} .
$$

Combining all these estimates, we obtain

$$
\sum_{1 \leq n \leq x} \rho(n)=x L(1, \chi)+R
$$

with

$$
|R| \leq 4 \Theta x^{1 / 2} z^{1 / 2}+\Theta x^{1 / 2} z^{1 / 2}+y+3 \Theta x y^{-1 / 2}=8 \Theta x y^{-1 / 2}+y .
$$

We set the "optimal" $y=4 \Theta^{2 / 3} x^{2 / 3}$. Our assumption $x \geq 10^{4} m^{3 / 8} \log m$ implies that indeed $1 \leq y<x$. We obtain

$$
|R| \leq 8 \Theta^{2 / 3} x^{2 / 3}<50 m^{1 / 8}(\log m)^{1 / 3} x^{2 / 3},
$$

as wanted.

The following lemma is the classical theorem of Tatuzawa [25].

Lemma 10.7. Let $0<\varepsilon<1 / 11.2$. Then, with at most one exception, for every positive integer $m>e^{1 / \varepsilon}$ the following holds. Let $\chi$ be a primitive real character $\bmod m$. Then $L(1, \chi)>0.655 \varepsilon m^{-\varepsilon}$.

Proof of Theorem 10.2. We assume that $m \geq 10^{160}$ is coarse and not the exceptional one from Lemma 10.7, where we set $\varepsilon=1 / 360$. Set $x=4 m^{1 / 2} / \log m$. We have $m>e^{360}$ and $x \geq 10^{4} m^{3 / 8} \log m$. Hence, combining Proposition 10.6 and Lemma 10.7 with $\varepsilon=1 / 360$, we obtain

$$
\sum_{1 \leq n \leq x} \rho(n) \geq \frac{0.655}{360} x m^{-1 / 360}-50 m^{1 / 8}(\log m)^{1 / 3} x^{2 / 3} .
$$


On the other hand, if $p$ is such that $\chi(p) \neq 1$ then

$$
\rho\left(p^{k}\right)=\frac{1-\chi(p)^{k+1}}{1-\chi(p)}=\left\{\begin{array}{l}
1, \chi(p)=0, \\
1, \chi(p)=-1,2 \mid k, \\
0, \chi(p)=-1,2 \nmid k .
\end{array}\right.
$$

Now assume that $\chi(p) \neq 1$ for all primes $p \leq x$. We have two cases.

If $m$ is prime, then, by (10.13) and the multiplicativity of $\rho(\cdot)$, for $1 \leq n<x$ we have $\rho(n)=1$ when $n$ is a full square, and $\rho(n)=0$ otherwise.

Now assume that $m$ is a product of two primes, $\ell$ being the smallest of them; in particular, $\rho(\ell)=1$. Since $x<m^{1 / 2}$, we have $\operatorname{gcd}(n, m) \in\{1, \ell\}$ for any $n$ in the range $1 \leq n<x$. It follows that $\rho(n)=1$ when $n$ is a full square or $\ell$ times a full square, and $\rho(n)=0$ otherwise.

Thus, in any case

$$
\sum_{1 \leq n \leq x} \rho(n) \leq 2 x^{1 / 2}
$$

Together with (10.12) this implies $m^{7 / 180} \leq 30000(\log m)^{2 / 3}$. This inequality is contradictory for $m \geq 10^{160}$.

Remark 10.8. At present, numerical refinements of Tatuzawa's theorem are available, see [11] and the references therein. In particular, using the main result of [11], one can reduce the numerical bound in Theorem 10.2 to $10^{140}$.

\section{The quantities $h(\Delta), \rho(\Delta)$ and $N(\Delta)$}

This section is preparatory for the "signature theorem", proved in Section 12 As before, $\Delta$ denotes a trinomial discriminant unless the contrary is stated explicitly. The following three quantities will play a crucial role in the sequel:

- $h(\Delta)$, the class number;

- $\rho(\Delta)$, the largest absolute value of a non-dominant singular modulus of discriminant $\Delta$

- $N(\Delta)$, the absolute norm $\left|\mathcal{N}_{\mathbb{Q}(x) / \mathbb{Q}}(x)\right|$, where $x$ is a singular modulus of discriminant $\Delta$; it clearly depends only on $\Delta$ and not on the particular choice of $x$.

For an arbitrary (not necessarily trinomial) discriminant we have upper estimates

$$
h(\Delta) \leq \pi^{-1}|\Delta|^{1 / 2}(2+\log |\Delta|), \quad \rho(\Delta) \leq e^{\pi|\Delta|^{1 / 2} / 2}+2079
$$

(for the first one see, for instance, Theorems 10.1 and 14.3 in [13, Chapter 12]). It turns out that for trinomial discriminants one can do much better. 
Proposition 11.1. Let $\Delta$ be a trinomial discriminant. Then

$$
101 \leq h(\Delta)<3|\Delta|^{1 / 2} / \log |\Delta|, \quad 700|\Delta|^{-3} \leq \rho(\Delta)<|\Delta|^{0.8} .
$$

An immediate application is the following much sharper form of Corollary 4.2 (the "principal inequality").

Corollary 11.2 (refined "principal inequality"). Let $\Delta$ be a trinomial discriminant of signature $(m, n)$ and $x_{1}, x_{2}$ non-dominant singular moduli of discriminant $\Delta$. Then

$$
\begin{aligned}
\left|1-\left(x_{2} / x_{1}\right)^{n}\right| & \leq e^{(m-n)\left(-\pi|\Delta|^{1 / 2}+\log |\Delta|\right)}, \\
|| x_{1}|-| x_{2}|| & \leq \rho(\Delta) e^{(m-n)\left(-\pi|\Delta|^{1 / 2}+\log |\Delta|\right)}, \\
|| x_{1}|-| x_{2}|| & \leq \rho(\Delta) e^{-\pi|\Delta|^{1 / 2}+\log |\Delta|}, \\
|| x_{1}|-| x_{2}|| & \leq e^{-\pi|\Delta|^{1 / 2}+2 \log |\Delta|} .
\end{aligned}
$$

For subsequent applications we need to estimate the product $h(\Delta) \log \rho(\Delta)$. Proposition 11.1 implies the estimate

$$
-9|\Delta|^{1 / 2}<h(\Delta) \log \rho(\Delta) \leq 2.4|\Delta|^{1 / 2} .
$$

However, this is insufficient for us: we need an estimate of the shape $o\left(|\Delta|^{1 / 2}\right)$ on both sides.

Proposition 11.3. Let $\Delta$ be a trinomial discriminant. Then

$$
-31|\Delta|^{1 / 2} / \log |\Delta|<h(\Delta) \log \rho(\Delta)<120|\Delta|^{1 / 2} / \log |\Delta| .
$$

Finally, we need an estimate for $N(\Delta)$. It is known that $N(\Delta)>1$ for any discriminant $\Delta$, see [2, 18]. For trinomial discriminants one can say much more.

Proposition 11.4. Let $\Delta$ be a trinomial discriminant. Then

$$
\log N(\Delta)=\pi|\Delta|^{1 / 2}+(h(\Delta)-1) \log \rho(\Delta)+O_{1}\left(e^{-\pi|\Delta|^{1 / 2}+2 \log |\Delta|}\right) .
$$

In particular,

$$
\log N(\Delta)>\pi|\Delta|^{1 / 2}-32|\Delta|^{1 / 2} / \log |\Delta| .
$$

Propositions 11.1, 11.3 and 11.4 are proved in the subsequent subsections. Since $\Delta$ is fixed, we may omit it in the sequel and write simply $h, \rho$ and $N$.

\subsection{Proof of Proposition 11.1}

According to Proposition 8.1, all suitable integers except 1 are prime numbers not exceeding $|\Delta / 3|^{1 / 2}$. Each of them occurs in most 2 triples $(a, b, c) \in T_{\Delta}$ (see the proof of Proposition 3.4). Hence $h \leq 1+2 \pi\left(|\Delta / 3|^{1 / 2}\right)$. Theorem 2 in [23, page 69] states that

$$
\frac{X}{\log X-1 / 2} \leq \pi(X) \leq \frac{X}{\log X-3 / 2} \quad(X \geq 67)
$$


Since $|\Delta| \geq 10^{11}$, we can apply this with $X=|\Delta / 3|^{1 / 2}$. We obtain

$$
h \leq 1+4 \frac{|\Delta / 3|^{1 / 2}}{\log |\Delta / 3|-3}<3 \frac{|\Delta|^{1 / 2}}{\log |\Delta|},
$$

which proves the upper estimate for $h$.

The lower bound for $h$ follows from the work of Watkins [29, which implies that a fundamental discriminant $\Delta$ with $h(\Delta) \leq 100$ satisfies $|\Delta| \leq 2383747$ : see Table 4 on page 936 of $\left[29\right.$. But $|\Delta| \geq 10^{11}$, a contradiction.

Now let $x$ be a non-dominant singular modulus of discriminant $\Delta$ and $a$ the corresponding suitable integer. Since $x$ is not dominant, we have $a>1$, which implies that $a>4|\Delta|^{1 / 2} / \log |\Delta|$. Hence

$$
|x| \leq e^{\pi|\Delta|^{1 / 2} / a}+2079<e^{(\pi / 4) \log |\Delta|}+2079<|\Delta|^{0.8},
$$

which proves the upper estimate for $\rho$.

As for the lower estimate $\rho \geq 700|\Delta|^{-3}$, it holds true for any discriminant $\Delta \neq-3$, not only trinomial discriminants, due to the following lemma.

Lemma 11.5. Let $x$ be a singular modulus of discriminant $\Delta \neq-3$ (not necessarily trinomial). Then $|x| \geq 700|\Delta|^{-3}$.

For the proof see [1, Corollary 5.3].

\subsection{Proof of Proposition 11.3; the upper estimate}

Let $1<a_{1}<\ldots<a_{k}$ be all suitable integers for $\Delta$. Since 1 occurs in one triple from $T_{\Delta}$ and each $a_{i}$ in at most two triples, we have

$$
h \leq 2 k+1 \leq 2 \pi\left(a_{k}\right)+1
$$

Now note that $a_{k} \leq 5 a_{1}$ by Proposition 5.5, and $a_{1} \geq 10^{4}$, see Remark 8.2. Hence we may use (11.4) with $X=5 a_{1}$. We obtain

$$
h \leq 2 \frac{5 a_{1}}{\log \left(5 a_{1}\right)-3 / 2}+1<10 \frac{a_{1}}{\log a_{1}} .
$$

Now, using that $4|\Delta|^{1 / 2} / \log |\Delta|<a_{1}<|\Delta / 3|^{1 / 2}$, we obtain

$$
h \log \rho<10 \frac{a_{1}}{\log a_{1}} \log \left(e^{\pi|\Delta|^{1 / 2} / a_{1}}+2079\right)<50 \frac{|\Delta|^{1 / 2}}{\log a_{1}}<120 \frac{|\Delta|^{1 / 2}}{\log |\Delta|},
$$

as wanted.

(Note that our argument is quite loose: the numerical constant 120 can be easily improved.) 


\subsection{Proof of Proposition 11.3; the lower estimate}

We need a lemma. Recall (see Section 2) that $\mathcal{F}$ denotes the standard fundamental domain, and that

$$
\zeta_{3}=\frac{-1+\sqrt{-3}}{2}, \quad \zeta_{6}=\frac{1+\sqrt{-3}}{2} .
$$

Lemma 11.6. For $z \in \mathcal{F}$ the following is true.

1. If $\min \left\{\left|z-\zeta_{3}\right|,\left|z-\zeta_{6}\right|\right\} \geq 10^{-3}$ then $|j(z)| \geq 4.4 \cdot 10^{-5}$.

2. If $\min \left\{\left|z-\zeta_{3}\right|,\left|z-\zeta_{6}\right|\right\} \leq 10^{-3}$ then

$$
|j(z)| \geq 44000 \min \left\{\left|z-\zeta_{3}\right|,\left|z-\zeta_{6}\right|\right\}^{3} .
$$

The proof can be found in 4, Proposition 2.2].

Now we are ready to prove the lower estimate from Proposition 11.3. We consider two cases. Assume first that there exists $(a, b, c) \in T_{\Delta}$ with $a>1$ such that $\tau=(b+\sqrt{\Delta}) / 2 a$ satisfies

$$
\min \left\{\left|\tau-\zeta_{3}\right|,\left|\tau-\zeta_{6}\right|\right\} \geq 10^{-3}
$$

Lemma 11.6 implies that the non-dominant singular modulus $x=j(\tau)$ satisfies $|x| \geq 4.4 \cdot 10^{-5}$. Hence $\rho \geq 4.4 \cdot 10^{-5}$. Using Proposition 11.1 we obtain

$$
h \log \rho \geq 3 \frac{|\Delta|^{1 / 2}}{\log |\Delta|} \log \left(4.4 \cdot 10^{-5}\right)>-31 \frac{|\Delta|^{1 / 2}}{\log |\Delta|},
$$

as wanted.

Now assume that for every $(a, b, c) \in T_{\Delta}$ with $a>1$ the number $\tau$ as above satisfies $\min \left\{\left|\tau-\zeta_{3}\right|,\left|\tau-\zeta_{6}\right|\right\}<10^{-3}$. Let $\varepsilon$ be the smallest real number such that

$$
\min \left\{\left|\tau-\zeta_{3}\right|,\left|\tau-\zeta_{6}\right|\right\} \leq \varepsilon
$$

for every $\tau$ like this. In particular, $0<\varepsilon<10^{-3}$.

Lemma 11.6 implies that

$$
\rho \geq 44000 \varepsilon^{3}
$$

Now let us estimate $h$. Note that for $\tau=(b+\sqrt{\Delta}) / 2 a$ we have

$$
\operatorname{Im}\left(\tau-\zeta_{3}\right)=\operatorname{Im}\left(\tau-\zeta_{6}\right)=\frac{|\Delta|^{1 / 2}}{2 a}-\frac{\sqrt{3}}{2} .
$$

Hence every suitable $a>1$ satisfies

$$
\frac{\sqrt{3}}{2}<\frac{|\Delta|^{1 / 2}}{2 a} \leq \frac{\sqrt{3}}{2}+\varepsilon
$$

which can be rewritten as

$$
\frac{|\Delta|^{1 / 2}}{\sqrt{3}+2 \varepsilon} \leq a<\frac{|\Delta|^{1 / 2}}{\sqrt{3}}
$$


Since all such $a$ are prime and each occurs in at most 2 triples $(a, b, c) \in T_{\Delta}$, we have

$$
h \leq 2\left(\pi\left(\frac{|\Delta|^{1 / 2}}{\sqrt{3}}\right)-\pi\left(\frac{|\Delta|^{1 / 2}}{\sqrt{3}+2 \varepsilon}\right)\right)+3 .
$$

(We have to add 3 rather than 1 because $|\Delta|^{1 / 2} /(\sqrt{3}+2 \varepsilon)$ can accidentally be a prime number.) Using (11.4), we estimate

$$
\begin{aligned}
\pi\left(\frac{|\Delta|^{1 / 2}}{\sqrt{3}}\right) & \leq \frac{2}{\sqrt{3}} \frac{|\Delta|^{1 / 2}}{\log |\Delta|-\log 3-3} \\
& <\frac{2}{\sqrt{3}} \frac{|\Delta|^{1 / 2}}{\log |\Delta|}+6 \frac{|\Delta|^{1 / 2}}{(\log |\Delta|)^{2}}, \\
\pi\left(\frac{|\Delta|^{1 / 2}}{\sqrt{3}+2 \varepsilon}\right) & \geq \frac{2}{\sqrt{3}+2 \varepsilon} \frac{|\Delta|^{1 / 2}}{\log |\Delta|-\log 3-1} \\
& >\left(\frac{2}{\sqrt{3}}-\frac{4 \varepsilon}{3}\right) \frac{|\Delta|^{1 / 2}}{\log |\Delta|}-2 \frac{|\Delta|^{1 / 2}}{(\log |\Delta|)^{2}},
\end{aligned}
$$

which implies that

$$
\begin{aligned}
h & <\frac{8 \varepsilon}{3} \frac{|\Delta|^{1 / 2}}{\log |\Delta|}+9 \frac{|\Delta|^{1 / 2}}{(\log |\Delta|)^{2}}, \\
h \log \rho & >\left(\frac{8 \varepsilon}{3} \frac{|\Delta|^{1 / 2}}{\log |\Delta|}+9 \frac{|\Delta|^{1 / 2}}{(\log |\Delta|)^{2}}\right) \log \left(44000 \varepsilon^{3}\right)
\end{aligned}
$$

We will estimate each of the terms

$$
\frac{8 \varepsilon}{3} \frac{|\Delta|^{1 / 2}}{\log |\Delta|} \log \left(44000 \varepsilon^{3}\right), \quad 9 \frac{|\Delta|^{1 / 2}}{(\log |\Delta|)^{2}} \log \left(44000 \varepsilon^{3}\right)
$$

separately.

The function $\varepsilon \mapsto \varepsilon \log \left(44000 \varepsilon^{3}\right)$ is strictly decreasing on $\left(0,10^{-3}\right]$, which implies that

$$
\frac{8 \varepsilon}{3} \frac{|\Delta|^{1 / 2}}{\log |\Delta|} \log \left(44000 \varepsilon^{3}\right) \geq \frac{8 \cdot 10^{-3}}{3} \frac{|\Delta|^{1 / 2}}{\log |\Delta|} \log \left(4.4 \cdot 10^{-5}\right)>-\frac{|\Delta|^{1 / 2}}{\log |\Delta|} .
$$

To estimate the second term, note that, since $a<|\Delta / 3|^{1 / 2}$, we have

$$
\varepsilon \geq \frac{|\Delta|^{1 / 2}}{2 a}-\frac{\sqrt{3}}{2}=\frac{|\Delta|-3 a^{2}}{2 a\left(|\Delta|^{1 / 2}+a \sqrt{3}\right)} \geq \frac{1}{2 a\left(|\Delta|^{1 / 2}+a \sqrt{3}\right)}>\frac{\sqrt{3}}{4|\Delta|} .
$$

Hence $\log \left(44000 \varepsilon^{3}\right)>-3 \log |\Delta|$, and

$$
9 \frac{|\Delta|^{1 / 2}}{(\log |\Delta|)^{2}} \log \left(44000 \varepsilon^{3}\right)>-27 \frac{|\Delta|^{1 / 2}}{(\log |\Delta|)} .
$$

This proves that

$$
h \log \rho>-28 \frac{|\Delta|^{1 / 2}}{\log |\Delta|},
$$

better than wanted. 


\subsection{Proof of Proposition 11.4}

Let $x_{0}, x_{1}, \ldots, x_{h-1}$ be the singular moduli of discriminant $\Delta$, with $x_{0}$ dominant. Then

$$
\begin{aligned}
& \log \left(1-e^{-\pi|\Delta|^{1 / 2}+\log 2079}\right) \leq \log \left|x_{0}\right|-\pi|\Delta|^{1 / 2} \leq \log \left(1+e^{-\pi|\Delta|^{1 / 2}+\log 2079}\right), \\
& \log \left(1-e^{-\pi|\Delta|^{1 / 2}+\log |\Delta|}\right) \leq \log \left|x_{i}\right|-\log \rho \leq 0 \quad(i=1, \ldots, h-1),
\end{aligned}
$$

where the second inequality follows from Corollary 11.2 .

Summing up, we obtain

$$
\log N(\Delta)=\pi|\Delta|^{1 / 2}+(h-1) \log \rho+O_{1}\left(h\left|\log \left(1-e^{-\pi|\Delta|^{1 / 2}+\log |\Delta|}\right)\right|\right) .
$$

Since $|\Delta| \geq 10^{11}$ and $h \leq 3|\Delta|^{1 / 2} / \log |\Delta|$, we may bound the error term by $e^{-\pi|\Delta|^{1 / 2}+2 \log |\Delta|}$, which proves (11.2). And (11.3) follows from (11.2) and Proposition 11.3.

\section{The signature theorem}

In this section we prove Theorem 1.5. Let us reproduce the statement here.

Theorem 12.1. Let $\Delta$ be a trinomial discriminant of signature $(m, n)$. Assume that $|\Delta| \geq 10^{40}$. Then $m-n \leq 2$.

As before, in this section we assume that $\Delta$ is a trinomial discriminant. In particular, $\Delta$ is odd, square-free and has at most 2 prime divisors. We also use the notation $h, \rho, N$, etc. from Section 11 Throughout this section $L$ is the Hilbert Class Field of $\mathbb{Q}(\sqrt{\Delta})$. It is an unramified abelian extension of $\mathbb{Q}(\sqrt{\Delta})$ of degree $h$, generated over $\mathbb{Q}(\sqrt{\Delta})$ by any singular modulus of discriminant $\Delta$. It is also Galois over $\mathbb{Q}$, of degree $2 h$. Denoting

$$
H=\operatorname{Gal}(L / \mathbb{Q}(\sqrt{\Delta})), \quad G=\operatorname{Gal}(L / \mathbb{Q}),
$$

we have $G=H \cup H \iota$, where $\iota \in G$ denotes the complex conjugation. Note that $\sigma \iota=\iota \sigma^{-1}$ for every $\sigma \in H$; see, for instance, [12, Lemma 9.3].

We denote by $\mathcal{N}(\cdot)$ the absolute norm on $L$; that is, for $y \in L$ we set

$$
\mathcal{N}(y)=\left|\mathcal{N}_{L / \mathbb{Q}}(y)\right|=\prod_{\sigma \in G}\left|y^{\sigma}\right|=\prod_{\sigma \in H}\left|y^{\sigma}\right|^{2} .
$$

If $x$ is a singular modulus of discriminant $\Delta$ then $\mathcal{N}(x)=N(\Delta)^{2}$. Indeed, $N(\Delta)=\left|\mathcal{N}_{\mathbb{Q}(x) / \mathbb{Q}}(x)\right|$, and $\mathbb{Q}(x)$ is a subfield of $L$ of degree 2 .

The strategy is as follows. We introduce a certain non-zero algebraic integer $z \in L$ and estimate $\mathcal{N}(z)$ from above using the "principal inequality" as given in Corollary 11.2. Compared with the trivial lower estimate $\mathcal{N}(z) \geq 1$, this would imply the following weaker version of Theorem 12.1 when $|\Delta|$ is large we have $m-n \leq 4$. 
Using this, and applying Proposition 4.4 we obtain a non-trivial lower bound for $\mathcal{N}(z)$. Comparing it with the previously obtained upper bound, we prove Theorem 12.1 .

We start with some lemmas.

Lemma 12.2. Let $\Delta$ be a trinomial discriminant and $x_{1}, \ldots, x_{4}$ distinct singular moduli of discriminant $\Delta$. Then $x_{1} x_{2} \neq x_{3} x_{4}$.

Proof. Applying Galois conjugation, we may assume that $x_{1}$ is dominant. Then neither of $x_{2}, x_{3}, x_{4}$ is. From Proposition 11.1 and Lemma 11.5, we have

$$
\left|x_{2}\right| \geq 700|\Delta|^{-3}, \quad\left|x_{3}\right|,\left|x_{4}\right|<|\Delta|^{0.8} .
$$

It follows that

$$
e^{\pi|\Delta|^{1 / 2}}-2079<\left|x_{1}\right|=\left|x_{2}^{-1} x_{3} x_{4}\right|<|\Delta|^{4.6} / 700,
$$

which is impossible for $|\Delta| \geq 10^{11}$.

Lemma 12.3. A trinomial $t^{m}+A t^{n}+B \in \mathbb{R}[t]$ may have at most 4 real roots.

Proof. The derivative $m t^{m-1}+n A t^{n-1}$ may have at most 3 real roots, and the result follows by the Theorem of Rolle.

Alternatively, one may use the "Descartes' rule of signs", which implies that the trinomial may have at most 2 positive and at most 2 negative roots.

Since $h(\Delta) \geq 101>6$ by Proposition 11.1. Lemma 12.3 implies that there must exist at least 3 non-real singular moduli of discriminant $\Delta$. In particular, there exist two singular moduli $x_{1}, x_{2} \notin \mathbb{R}$ such that $x_{1} \neq x_{2}$ and $\bar{x}_{1} \neq x_{2}$. (We denote by $\bar{x}$ the complex conjugate of $x$.) Thus, $x_{1}, \bar{x}_{1}, x_{2}, \bar{x}_{2}$ are distinct nondominant singular moduli of discriminant $\Delta$. We set

$$
z=x_{1} \bar{x}_{1}-x_{2} \bar{x}_{2}=\left|x_{1}\right|^{2}-\left|x_{2}\right|^{2} .
$$

This $z$ is a non-zero (by Lemma 12.2) real algebraic integer.

Proposition 12.4. Let $\Delta$ be a trinomial discriminant of signature $(m, n)$. Then

$$
\log \mathcal{N}(z) \leq \pi|\Delta|^{1 / 2}(8-2(m-n))+2(m-n) \log |\Delta|+243|\Delta|^{1 / 2} / \log |\Delta|
$$

Proof. Using Corollary 11.2, we estimate

$$
|z|<2 \rho^{2} e^{(m-n)\left(-\pi|\Delta|^{1 / 2}+\log |\Delta|\right)} .
$$

Let us split $G$ into three subsets.

1. For $\sigma=$ id or $\sigma=\iota$ we have $z^{\sigma}=z$. Hence in these two cases

$$
\left|z^{\sigma}\right|<2 \rho^{2} e^{(m-n)\left(-\pi|\Delta|^{1 / 2}+\log |\Delta|\right)} .
$$


2. For every singular modulus $x$ of discriminant $\Delta$ there exists exactly one element $\sigma \in H$ such that $x^{\sigma}$ is dominant. We claim that $\bar{x}^{\sigma^{-1}}$ is then dominant as well. Indeed, since $x^{\sigma} \in \mathbb{R}$ (the dominant singular modulus is real), we have $\bar{x}^{\sigma^{-1}}=x^{\iota \sigma^{-1}}=x^{\sigma \iota}=x^{\sigma}$, as wanted.

Now let $\sigma_{1}, \sigma_{2} \in H$ be such that $x_{1}^{\sigma_{1}}$ is dominant, and so is $x_{2}^{\sigma_{2}}$. Then there exist exactly 8 elements $\sigma \in G$ such that one of $x_{1}^{\sigma}, \bar{x}_{1}^{\sigma}, x_{2}^{\sigma}, \bar{x}_{2}^{\sigma}$ is dominant: they are

$$
\sigma_{1}, \sigma_{1} \iota, \sigma_{1}^{-1}, \sigma_{1}^{-1} \iota, \sigma_{2}, \sigma_{2} \iota, \sigma_{2}^{-1}, \sigma_{2}^{-1} \iota .
$$

For these $\sigma$ we have the upper estimate

$$
\left|z^{\sigma}\right|<\rho\left(e^{\pi|\Delta|^{1 / 2}}+2079\right)+\rho^{2}<2 \rho e^{\pi|\Delta|^{1 / 2}} .
$$

3. For the remaining $2 h-10$ elements $\sigma \in G$ none of $x_{1}^{\sigma}, \bar{x}_{1}^{\sigma}, x_{2}^{\sigma}, \bar{x}_{2}^{\sigma}$ is dominant. Hence for those $\sigma$ we have

$$
\left|z^{\sigma}\right| \leq 2 \rho^{2}
$$

It follows that

$$
\begin{aligned}
\mathcal{N}(z) & <\left(2 \rho^{2} e^{(m-n)\left(-\pi|\Delta|^{1 / 2}+\log |\Delta|\right)}\right)^{2}\left(2 \rho e^{\pi|\Delta|^{1 / 2}}\right)^{8}\left(2 \rho^{2}\right)^{2 h-10} \\
& \leq e^{\pi|\Delta|^{1 / 2}(8-2(m-n))+4 h \log \rho^{*}+2 h \log 2+2(m-n) \log |\Delta|}
\end{aligned}
$$

where $\rho^{*}=\max \{\rho, 1\}$. Using Propositions 11.1 and 11.3, we estimate

$$
2 h \log \rho^{*}+h \log 2 \leq 243|\Delta|^{1 / 2} / \log |\Delta| .
$$

Whence the result.

Corollary 12.5. We have either $|\Delta|<10^{30}$ or $m-n \leq 4$.

Proof. Since $\mathcal{N}(z) \geq 1$, Proposition 12.4 implies that

$$
(m-n)\left(1-\frac{\log |\Delta|}{\pi|\Delta|^{1 / 2}}\right)-4 \leq \frac{130|\Delta|^{1 / 2} / \log |\Delta|}{\pi|\Delta|^{1 / 2}} .
$$

Hence

$$
m-n<\left(4+\frac{50}{\log |\Delta|}\right)\left(1-\frac{\log |\Delta|}{\pi|\Delta|^{1 / 2}}\right)^{-1} .
$$

When $|\Delta| \geq 10^{30}$ this implies that $m-n<4$.8. Hence $m-n \leq 4$, as wanted.

To improve on this, we need a non-trivial lower estimate for $\mathcal{N}(z)$.

Proposition 12.6. Assume that $|\Delta| \geq 10^{30}$. Then $\log \mathcal{N}(z)>3.36 \log N(\Delta)$. 
Proof. For every rational prime number $p$ we want to estimate $\nu_{p}(\mathcal{N}(z))$ from below. Let $\mathfrak{p} \mid p$ be an $L$-prime above $p$ and $e_{p}$ the ramification index of $p$ in $L$. Note that

$$
e_{p}= \begin{cases}1, & p \nmid \Delta, \\ 2, & p \mid \Delta,\end{cases}
$$

because $L$ is unramified over $\mathbb{Q}(\sqrt{\Delta})$. We denote by $\nu_{\mathfrak{p}}(\cdot)$ the $\mathfrak{p}$-adic valuation and define $\nu_{\mathfrak{p}}^{\prime}(\cdot)=\nu_{\mathfrak{p}}(\cdot) / e_{p}$. Then, clearly, $\nu_{p}(m)=\nu_{\mathfrak{p}}^{\prime}(m)$ for any $m \in \mathbb{Z}$.

Proposition 4.3 implies that the set

$$
S_{p}=S_{p}(\Delta)=\left\{\nu_{\mathfrak{p}}^{\prime}(x): x \text { singular modulus of discriminant } \Delta\right\} .
$$

may consist of at most 2 elements. In case it consists of just one element, this element is $\nu_{\mathfrak{p}}^{\prime}(N) / h=\nu_{p}(N) / h$, where, as before, we use notation $h=h(\Delta)$ and $N=N(\Delta)$. Hence for every $\sigma \in G$ we have

$$
\nu_{\mathfrak{p}}^{\prime}\left(x_{1}^{\sigma}\right)=\nu_{\mathfrak{p}}^{\prime}\left(\bar{x}_{1}^{\sigma}\right)=\nu_{\mathfrak{p}}^{\prime}\left(x_{2}^{\sigma}\right)=\nu_{\mathfrak{p}}^{\prime}\left(\bar{x}_{2}^{\sigma}\right)=\nu_{p}(N) / h,
$$

which implies that $\nu_{\mathfrak{p}}^{\prime}\left(z^{\sigma}\right) \geq 2 \nu_{p}(N) / h$. Therefore in this case

$$
\nu_{p}(\mathcal{N}(z))=\nu_{\mathfrak{p}}^{\prime}(\mathcal{N}(z)) \geq 2 h \cdot 2 \nu_{p}(N) / h=4 \nu_{p}(N) .
$$

Now assume that $S_{p}$ consists of 2 distinct elements: $S_{p}=\left\{\alpha_{p}, \beta_{p}\right\}$, with

$$
\alpha_{p}<\nu_{p}(N) / h<\beta_{p} .
$$

Since $m-n \leq 4$, Proposition 4.4 implies that at most 4 singular moduli of discriminant $\Delta$ have $\nu_{\mathfrak{p}}^{\prime}$-valuation $\alpha_{p}$. It follows that there exist at most 32 elements $\sigma \in G$ such that one of $x_{1}^{\sigma}, \bar{x}_{1}^{\sigma}, x_{2}^{\sigma}, \bar{x}_{2}^{\sigma}$ has $\nu_{\mathfrak{p}}^{\prime}$-valuation $\alpha_{p}$. For the remaining $2 h-32$ elements $\sigma \in G$ we have

$$
\nu_{\mathfrak{p}}^{\prime}\left(x_{1}^{\sigma}\right)=\nu_{\mathfrak{p}}^{\prime}\left(\bar{x}_{1}^{\sigma}\right)=\nu_{\mathfrak{p}}^{\prime}\left(x_{2}^{\sigma}\right)=\nu_{\mathfrak{p}}^{\prime}\left(\bar{x}_{2}^{\sigma}\right)=\beta_{p}>\nu_{p}(N) / h,
$$

which implies that for these $\sigma$ we have $\nu_{\mathfrak{p}}^{\prime}\left(z^{\sigma}\right)>2 \nu_{p}(N) / h$. Therefore

$$
\nu_{p}(\mathcal{N}(z))>(2 h-32) \cdot 2 \nu_{p}(N) / h>3.36 \nu_{p}(N),
$$

where the last inequality follows from $h \geq 101$.

Thus, for every $p$ we have either (12.5) or (12.6). This proves the wanted lower bound $\log \mathcal{N}(z)>3.36 \log N$.

We are now ready to prove Theorem 12.1

Proof of Theorem 12.1. Combining the lower estimate from Propositions 12.6 with (11.3), we obtain

$$
\log \mathcal{N}(z) \geq 3.36 \pi|\Delta|^{1 / 2}-110|\Delta|^{1 / 2} / \log |\Delta| .
$$

Comparing this with the upper estimate from Proposition 12.4, we obtain

$$
\pi|\Delta|^{1 / 2}(4.64-2(m-n))+8 \log |\Delta|+360|\Delta|^{1 / 2} / \log |\Delta|>0 .
$$

If $m-n \geq 3$ then this implies that $|\Delta|<10^{40}$ after a trivial calculation. 


\section{References}

[1] Yuri Bilu, Bernadette Faye, and Huilin Zhu, Separating singular moduli and the primitive element problem, Q. J. Math. 71 (2020), no. 4, 1253-1280. MR 4186519

[2] Yuri Bilu, Philipp Habegger, and Lars Kühne, No singular modulus is a unit, Int. Math. Res. Not. IMRN (2020), no. 24, 10005-10041. MR 4190395

[3] Yuri Bilu and Florian Luca, Trinomials with given roots, Indag. Math. (N.S.) 31 (2020), no. 1, 33-42. MR 4052259

[4] Yuri Bilu, Florian Luca, and Amalia Pizarro-Madariaga, Rational products of singular moduli, J. Number Theory 158 (2016), 397-410. MR 3393559

[5] Yuri Bilu, David Masser, and Umberto Zannier, An effective "theorem of André" for $C M$-points on a plane curve, Math. Proc. Cambridge Philos. Soc. 154 (2013), no. 1, 145-152. MR 3002589

[6] Andrew R. Booker, Quadratic class numbers and character sums, Math. Comp. 75 (2006), no. 255, 1481-1492. MR 2219039

[7] Matteo Bordignon, Partial Gaussian sums and the Pólya-Vinogradov inequality for primitive characters, arXiv:2001.05114 (2020).

[8] D. A. Burgess, On character sums and L-series, Proc. London Math. Soc. (3) 12 (1962), 193-206. MR 0132733

[9], On character sums and L-series. II, Proc. London Math. Soc. (3) 13 (1963), 524-536. MR 0148626

[10] J. W. S. Cassels, Local Fields, London Mathematical Society Student Texts, vol. 3, Cambridge University Press, Cambridge, 1986. MR 861410

[11] Yong-Gao Chen, On the Siegel-Tatuzawa-Hoffstein theorem, Acta Arith. 130 (2007), no. 4, 361-367. MR 2365711

[12] David A. Cox, Primes of the Form $x^{2}+n y^{2}$, second ed., Pure and Applied Mathematics (Hoboken), John Wiley \& Sons, Inc., Hoboken, NJ, 2013, Fermat, class field theory, and complex multiplication. MR 3236783

[13] Loo Keng Hua, Introduction to Number Theory, Springer-Verlag, Berlin-New York, 1982, Translated from the Chinese by Peter Shiu. MR 665428

[14] Kenneth Ireland and Michael Rosen, A classical introduction to modern number theory, second ed., Graduate Texts in Mathematics, vol. 84, Springer-Verlag, New York, 1990. MR 1070716

[15] Henryk Iwaniec and Emmanuel Kowalski, Analytic Number Theory, American Mathematical Society Colloquium Publications, vol. 53, American Mathematical Society, Providence, RI, 2004. MR 2061214

[16] Niraek Jain-Sharma, Tanmay Khale, and Mengzhen Liu, Explicit burgess bound for composite moduli, Int. J. Number Theory, to appear, arXiv:2010.09530

[17] Youness Lamzouri, Xiannan Li, and Kannan Soundararajan, Conditional bounds for the least quadratic non-residue and related problems, Math. Comp. 84 (2015), no. 295, 2391-2412. MR 3356031

[18] Yingkun Li, Singular units and isogenies between CM elliptic curves, Compos. Math. 157 (2021), no. 5, 1022-1035. MR 4251608

[19] Florian Luca and Antonin Riffaut, Linear independence of powers of singular moduli of degree three, Bull. Aust. Math. Soc. 99 (2019), no. 1, 42-50. MR 3896878

[20] Edouard Lucas, Theorie des Fonctions Numeriques Simplement Periodiques, Amer. J. Math. 1 (1878), no. 4, 289-321. MR 1505176

[21] Paul Pollack, Bounds for the first several prime character nonresidues, Proc. Amer. Math. Soc. 145 (2017), no. 7, 2815-2826. MR 3637932 
[22] Antonin Riffaut, Equations with powers of singular moduli, Int. J. Number Theory 15 (2019), no. 3, 445-468. MR 3925747

[23] J. Barkley Rosser and Lowell Schoenfeld, Approximate formulas for some functions of prime numbers, Illinois J. Math. 6 (1962), 64-94. MR 0137689

[24] Wolfgang M. Schmidt, Equations over Finite Fields. An Elementary Approach, Lecture Notes in Mathematics, Vol. 536, Springer-Verlag, Berlin-New York, 1976. MR 0429733

[25] Tikao Tatuzawa, On a theorem of Siegel, Jap. J. Math. 21 (1951), 163-178. MR 0051262

[26] The PARI Group, PARI/GP (Version 2.11.0), 2018, Bordeaux, http://pari.math.u-bordeaux.fr/

[27] The Sage Developers, Sagemath, the Sage Mathematics Software System (Version 8.6), 2019, https://www.sagemath.org

[28] Enrique Treviño, The Burgess inequality and the least kth power non-residue, Int. J. Number Theory 11 (2015), no. 5, 1653-1678. MR 3376232

[29] Mark Watkins, Class numbers of imaginary quadratic fields, Math. Comp. 73 (2004), no. 246, 907-938. MR 2031415 